\title{
TPN-associated intestinal epithelial cell atrophy is modulated by TLR4/EGF signaling pathways
}

\author{
Jennifer J. Freeman, ${ }^{, \dagger}{ }^{\dagger}$ Yongjia Feng, ${ }^{*}$ Farokh R. Demehri, $*$ Peter J. Dempsey, ${ }^{\ddagger}$ \\ and Daniel H. Teitelbaum*,+, \\ *Department of Surgery, Section of Pediatric Surgery, and ${ }^{\dagger}$ Center for Organogenesis, University of \\ Michigan, Ann Arbor, Michigan, USA; and ${ }^{\dagger}$ School of Medicine, Department of Pediatrics, University of \\ Colorado, Denver, Anschutz Medical Campus, Denver, Colorado, USA
}

\begin{abstract}
Recent studies suggest a close interaction between epidermal growth factor (EGF) and TLR signaling in the modulation of intestinal epithelial cell (IEC) proliferation; however, how these signaling pathways adjust IEC proliferation is poorly understood. We utilized a model of total parenteral nutrition (TPN), or enteral nutrient deprivation, to study this interaction as TPN results in mucosal atrophy due to decreased IEC proliferation and increased apoptosis. We identified the novel finding of decreased mucosal atrophy in TLR4 knockout (TLR4KO) mice receiving TPN. We hypothesized that EGF signaling is preserved in TLR4KO-TPN mice and prevents mucosal atrophy. $\mathrm{C} 57 \mathrm{Bl} / 6$ and strain-matched TLR4KO mice were provided either enteral feeding or TPN. IEC proliferation and apoptosis were measured. Cytokine and growth factor abundances were detected in both groups. To examine interdependence of these pathways, ErbB1 pharmacologic blockade was used. The marked decline in IEC proliferation with TPN was nearly prevented in TLR4KO mice, and intestinal length was partially preserved. EGF was significantly increased, and TNF- $\alpha$ decreased in TLR4KO-TPN versus wild-type (WT)TPN mice. Apoptotic positive crypt cells were 15-fold higher in WT-TPN versus TLR4KO-TPN mice. Bcl-2 was significantly increased in TLR4KO-TPN mice, while Bax decreased 10-fold. ErbB1 blockade prevented this otherwise protective effect in TLR4KO-sTPN mice. TLR4 blockade significantly prevented TPN-associated atrophy by preserving proliferation and preventing apoptosis. This is driven by a reduction in TNF- $\alpha$ abundance and increased EGF. Potential manipulation of this regulatory pathway may have significant clinical potential to prevent TPNassociated atrophy.-Freeman, J. J., Feng, Y., Demehri, F. R., Dempsey, P. J., Teitelbaum, D. H. TPN-associated intestinal epithelial cell atrophy is modulated by TLR4/ EGF signaling pathways. FASEB J. 29, 2943-2958 (2015). www.fasebj.org
\end{abstract}

Abbreviations: BrdU, 5-bromo-2-deoxyuridine; EBF, epithelial barrier function; EGF, epidermal growth factor; EGFR, epidermal growth factor receptor; HB-EGF, heparin-binding epidermal growth factor; IEC, intestinal epithelial cell; IF, immunofluorescence; NRG, neuroregulin; p-AKT, phosphorylated protein kinase B; PCNA, proliferating cell nuclear antigen; RT-PCR,

(continued on next page)
Key Words: apoptosis $\cdot$ epidermal growth factor $\cdot$ epithelial cell proliferation $\cdot$ mucosal atrophy $\cdot$ total parenteral nutrition

TOTAL PARENTERAL NUTRITION (TPN) is utilized for nutritional support for over 375,000 U.S. patients each year who are unable to tolerate enteral nutrition in either the short term as a result of postoperative gastrointestinal dysfunction or in the long term as a result of loss of intestinal length $(1,2)$. TPN, however, leads to mucosal atrophy due to decreased intestinal epithelial cell (IEC) proliferation and increased apoptosis, with resultant loss in mucosal epithelial barrier function $(\mathrm{EBF})$, decline in the size of the crypt/villus complex, and reduction in intestinal length (3-5). Using a mouse TPN model, we previously showed that mucosalderived epidermal growth factor (EGF) and its downstream signals are down-regulated while $\mathrm{TNF}-\alpha$ is up-regulated. Importantly, both changes contribute to an overall phenotype of mucosal atrophy (6).

An important signaling pathway which drives TNF- $\alpha$ expression is via TLR activation. In particular, activation of TLR4 via signaling through a MyD88/TRIF downstream signaling pathway can significantly up-regulate a number of proinflammatory cytokines, including TNF- $\alpha$. Interestingly, TLR4 signaling is markedly up-regulated in our TPN model in a MyD88-dependent fashion (6). In fact, our laboratory has shown that knocking out the TLR common adaptor MyD88 led to a prevention of TNF- $\alpha$ expression (7).

The interdependence of TLR signaling on IEC survival has been demonstrated previously. Sodhi et al. showed that when TLR4 is deleted in the IEC population, intestinal proinflammatory processes, such as a mouse necrotizing enterocolitis model, were prevented with resultant minimal cytokine elevation and preservation of small intestine mucosa (8).

It is well appreciated that ErbB1 is critical for mediating IEC proliferation and antiapoptotic actions (9, 10). Interestingly, a link between successful EGF/ErbB1 signaling and integrity of TNF- $\alpha$ signaling has been shown. Kaiser and Polk showed that TNF- $\alpha$ receptor (TNFR) 1 signaling inhibited proliferation, whereas TNF- $\alpha$ R2 (TNFR2) promoted proliferation (11). The same laboratory later

\footnotetext{
${ }^{1}$ Correspondence: Section of Pediatric Surgery, University of Michigan, Mott Children's Hospital, 1540 E Hospital Dr., SPC 4211, Ann Arbor, MI 48109-4211, USA. E-mail: dttlbm@ umich.edu

doi: $10.1096 /$ fj.14-269480
} 
showed that activation of TNFR1 signaling inhibited EGF stimulated proliferation by promoting internalization of EGFR (12). This interdependence of TNF- $\alpha$ signaling and EGF may have strong relevance to TPN-associated atrophy. Our laboratory has shown that EGF failed to drive effective IEC proliferation in an environment with increased TNFR1 expression and an absence of TNFR2 (6). Thus, EGF and TNFR1 appear to have distinct alternative roles in modulating IEC proliferation and apoptosis; however, it is not clear what factors may be driving the strong TNFR1 signaling with TPN administration.

A critical link between EGF and TLR signaling has also been observed to modulate IEC proliferation and apoptosis (10). Hsu et al. found that in the colon TLR4 activation up-regulated the EGF receptor ligands amphiregulin and epiregulin (13). The mechanisms driving such activation between TLR4 and EGF-signaling pathways has been reported. Fukata and Abreu described that TLR4 activation induces cyclooxygenase 2 and prostaglandin $\mathrm{E}_{2}$ production, which can then activate the EGF receptor (EGFR) pathway (14). However, despite these observations, the exact mechanisms and interrelations of these 2 important signaling pathways are not clear.

Because our TPN mouse model shows significant intestinal atrophy due to a loss of EGF and the presence of increased TLR abundance, this TPN model offers a useful approach to investigate the interaction of these pathways $(6,7)$. We hypothesized that EGF signaling is preserved in TLR4 knockout (TLR4KO)-TPN mice and may function to prevent mucosal atrophy.

The current work demonstrates for the first time that small intestinal architecture and barrier function are significantly altered without effective TLR4 signaling. It also demonstrates the novel finding that TPN-associated intestinal mucosal atrophy is prevented in TLR4KO mice as a result of sustained epithelial proliferation and reduced apoptosis. Loss of TLR4 signaling in this model also resulted in a preservation of EGF expression and decreased mucosal abundance of TNF- $\alpha$. The present work demonstrates the critical role TLR4 has on modulation of intestinal growth and suggests that strategies to modulate TLR4 signaling may be beneficial in patients receiving TPN.

\section{MATERIALS AND METHODS}

Animals

$\mathrm{C} 57 \mathrm{Bl} / 6$ male, specific-pathogen-free 9- to 10-week-old male mice (The Jackson Laboratory, Bar Harbor, ME, USA) were maintained under temperature-, humidity-, and light-controlled conditions. Body weight and age-matched male TLR4-knockout mice (TLR4KO; 007227, B6.B10ScN-Tlr4 $4^{\text {lps-del }} /$ JthJ; Jackson Laboratory) were also studied. The mice were initially fed standard mouse chow and water ad libitum and were allowed to acclimate for 1 week before surgery. During the administration of intravenous solutions, the mice were individually housed in metabolic cages to prevent coprophagia. The study protocol was

(continued from previous page)

real-time PCR; TLR4KO, TLR 4 knockout; TNFR, TNF- $\alpha$ receptor; TPN, total parenteral nutrition; TRAF6, TNF- $\alpha$ receptor-associated factor 6; WT, wild-type; ZO-1, zonula occludens-1 approved by University of Michigan Committee on the Use and Care of Animals (approval \#3986).

\section{Operative procedures and TPN delivery}

Cannulation and administration of TPN was identical with that previously described $(15,16)$. TPN mice received intravenous TPN at $4.8 \mathrm{ml}$ per day. Nitrogen and energy delivery was matched between groups (isonitrogenous/isocaloric). This TPN solution and the administration rate allow for adequate caloric delivery, matching the amount that the enterally fed mice received (16, 17). The sham treatment group consisted of enterally fed mice provided intravenous saline at the same rate $(4.8 \mathrm{ml}$ per day) as the infusions in the TPN group. Mice were euthanized with carbon dioxide 7 days after cannulation. Body weight was recorded on the day of cannulation and just before euthanasia. Mice were killed early if their health deteriorated to a level found to be unacceptable for their well-being and were not included in the final data analysis.

\section{EGF blockade}

For the pharmacologic EGF blockade, animals were provided the ErbB1 inhibitor gefitinib (LC Laboratories, Woburn, MA, USA; $2.5 \mathrm{mg} / \mathrm{ml}$ in $1 \%$ aqueous Tween- $80,200 \mu \mathrm{l}$ per dose) by oral gavage twice daily starting 3 days before cannulation and continued until animals were killed. As with other groups, mice were killed early if their health deteriorated to a level found to be unacceptable for their well-being and were not included in the final data analysis.

\section{Intestinal morphology assessment}

Villus length and crypt depth were measured in $\geq 10$ welloriented, full-length crypt-villus units per specimen and averaged. Data were analyzed with commercially available digital image analysis software (NIS-Elements, AR 3.0; Nikon, Melville, NY, USA).

\section{RNA isolation and quantitative real-time PCR (RT-PCR)}

RNA extraction from mucosal scrapings was performed according to published procedures $(18,19)$. qPCR was measured with a Rotor-Gene 6000 (Qiagen, Hilden, Germany), and $\beta$-actin or 18S R1 ribosomal RNA was used as the internal control for normalization. Table 1 lists the primer sequences.

\section{Western immunoblot analysis}

The isolation of IECs and Western blot analysis were performed as previously described (20) with primary antibodies including the following: mouse anti-Bax (1:200, sc-7480; Santa Cruz Biotechnology, Santa Cruz, CA, USA), mouse anti-Bcl-2 (1:200, sc7382; Santa Cruz Biotechnology), mouse anti-phospho-Iк $\beta \alpha$ (Ser32/36; 1:1000, 9246; Cell Signaling Technology, Danvers, MA, USA), mouse anti-Iк $\beta \alpha$ (L35A5 Amino terminal antigen; 1:1000, 4814; Cell Signaling Technology), mouse anti-phosphoERK (Thr202/Tyr204; 1:1000, 9106S; Cell Signaling Technology), rabbit anti-ERK1/2 (1:1000, 9102; Cell Signaling Technology), rabbit anti-phosphorylated protein kinase B 1/2/3 (p-AKT; Ser473 and Thr308; 1:200, sc-25658; Santa Cruz Biotechnology), mouse anti-proliferating cell nuclear antigen (PCNA; 1:3000, 
TABLE 1. RT-PCR primer sequences

\begin{tabular}{lll}
\hline \hline Mouse gene & \multicolumn{1}{c}{ Forward $\left(5^{\prime}-3^{\prime}\right)$} & \\
\hline Bcl-2 & ATGCCTTTGTGGACTATATGGC & Reverse $\left(5^{\prime}-\mathbf{3}^{\prime}\right)$ \\
Bax & TGAAGACAGGGGCCTTTTG & AATTCGCCGGAGACACTCG \\
Fas & GGTGGAAACAAACTGCACCC & ACAGTGTTCACAGCCAGGAG \\
FasL & ACCGCTCTGATCTCTGGAGT & GGCTGGTTGTTGAAGACTG \\
TNF- $\alpha$ & CCGGGAGAAGAGGGATAGCTTGG & TCGGACAGTCACTCACCAAGT \\
IFN- $\gamma$ & ATGAACGCTACACACTGCATC & CCATCCTTTTGCAGTTCCTC \\
TLR-2 & ACAATAGAGGGAGAGCCTT & AGTGTCTGGTAAGGATTTCCAT \\
TLR-5 & CTGCAACTGTGAACTTAGCA & ACTTTAGGGACCGCAT \\
TLR-7 & TGTCTCTTCCGTGTCCACAT & GGATGATCCTGGCCTATCTC \\
TLR-9 & CTGTACCAGGAGGGACAAGG & CAGTTTGTCAGAGGGAGCCT \\
IL-6 & TCTCTGGGAAATCGTGGAAA & GATGGTCTTGGTCCTTAGCC \\
IL-10 & ACCTGGTAGAAGTGATGCCCCCAGGCA & CTATGCAGTTGATGAAGATGTCAAA \\
EGF & TTCTCACAAGGAAAGAGCATCTC & GTCCTGTCCCGTTAAGGAAAC \\
EGFR & GCATCATGGGAGAGAACAACA & TCAGGAACCATTACTCCATAGGT \\
Amphiregulin & GGTCTTAGGCTCAGGCCATTA & CGCTTATGGTGGAAACCTCTC \\
HB-EGF & CGGGGAGTGCAGATACCTG & TTCTCCACTGGTAGAGTCAGC \\
Epiregulin & CTGCCTCTTGGGTCTTGACG & GCGGTACAGTTATCCTCGGATTC \\
$\beta-A c t i n$ & ATGGAGCCGGACAGAAAAGC & CTTGCCACTCAGGGAAGGA \\
18S ribosomal & ATCGACACTTCGAACGCACT & CACACGTCTGAACTTCGGGA \\
RNA & & \\
\hline
\end{tabular}

2586; Cell Signaling Technology), and mouse anti- $\beta$-actin (1:1000, sc-130300; Santa Cruz Biotechnology). The secondary antibody used was the horseradish peroxidase conjugate of either goat antimouse or goat anti-rabbit IgG (1:2000; Santa Cruz Biotechnology) as appropriate.

\section{IF microscopy}

Sections of jejunum $(5 \mu \mathrm{m})$ were embedded in paraffin and placed on slides by our pathology department. Immunofluorescence (IF) staining was performed (18) with primary antibodies including the following: mouse anti-PCNA (1:3000, 2586, Cell Signaling Technology), mouse anti-5-bromo-2-deoxyuridine (BrdU; 1:50, sc-56255; Santa Cruz Biotechnology), rabbit antioccludin (6:1000, ab31721; Abcam, Cambridge, MA, USA), mouse anti-claudin 2 (5:1000, 325600; Invitrogen, Carlsbad, CA, USA), and rabbit anti-zonula occludens-1 (ZO-1; 1:100, 617300; Invitrogen). Secondary antibodies were Alexa Fluor 488 goat antimouse IgG (1:500, A21121; Invitrogen) or Alexa Fluor 555 donkey anti-rabbit IgG (1:1000, A31572; Invitrogen) as appropriate. DAPI staining was performed with Prolong Gold antifade reagent with DAPI (P36935; Life Technologies, Carlsbad, CA, USA).

\section{IEC proliferation assessment}

IEC proliferation was assessed by IF staining of the markers PCNA and BrdU as previously described (21).

\section{IEC apoptosis assessment}

IEC apoptosis was assessed by TUNEL and caspase 3 staining as previously described (21).

\section{Ussing chamber and permeability studies}

Experiments were performed in modified Ussing chambers (Physiologic Instruments, San Diego, CA, USA) on jejunal segments as previously described (22). Transepithelial resistance was determined according to Ohm's law. Permeability of the jejunum was assessed by measuring mucosal-to-serosal permeation of FITC-dextran (FD4; average molecular weight, 3000-5000; Sigma-Aldrich, St. Louis, MO, USA) as previously described (23). Briefly, $150 \mu$ l FITC-dextran $(50 \mathrm{mg} / \mathrm{ml}$, diluted with fresh Krebs buffer) was added to the mucosal compartment after a 30-minute equilibration period. Then $500 \mu \mathrm{l}$ samples were removed from the serosal compartment every 30 minutes, and fluorescence was measured with a Synergy 2 multimode microplate reader (BioTek Instruments, Winooski, VT, USA) at an excitation wavelength of $492 \mathrm{~nm}$ and an emission wavelength of $515 \mathrm{~nm}$. Permeability was expressed as the percentage of mucosal-to-serosal clearance of FITC-dextran.

\section{Statistical analysis}

Data are expressed as means \pm SD. All experiments were performed with $>6$ mice per group. A preliminary power analysis determined that a minimum of 6 subjects per study group would be needed on the basis of previous variance data from proliferation assays, our primary outcome measure. Student's $t$ tests were used for comparing 2 groups. ANOVA with Tukey post hoc testing was used to compare multiple groups. Significance was defined as $P<0.05$.

\section{RESULTS}

\section{TLR4KO mice provided TPN show less intestinal atrophy compared to WT-TPN mice}

Enterally fed TLR4KO-sham mice had similar gross small bowel characteristics compared to wild-type (WT)-sham mice (Fig. 1A). In contrast, loss of TLR4 signaling led to profound changes with TPN administration. WT mice undergo a significant decrease in small intestinal length with TPN administration $(P<0.0001)$; however, TLR4KOTPN mice showed no significant loss in length (Fig. $1 A$ ). When evaluated as a percentage change in small intestinal length (compared to the sham intestinal length of each group), the TLR4KO-TPN mice had a 4-fold lower decline 

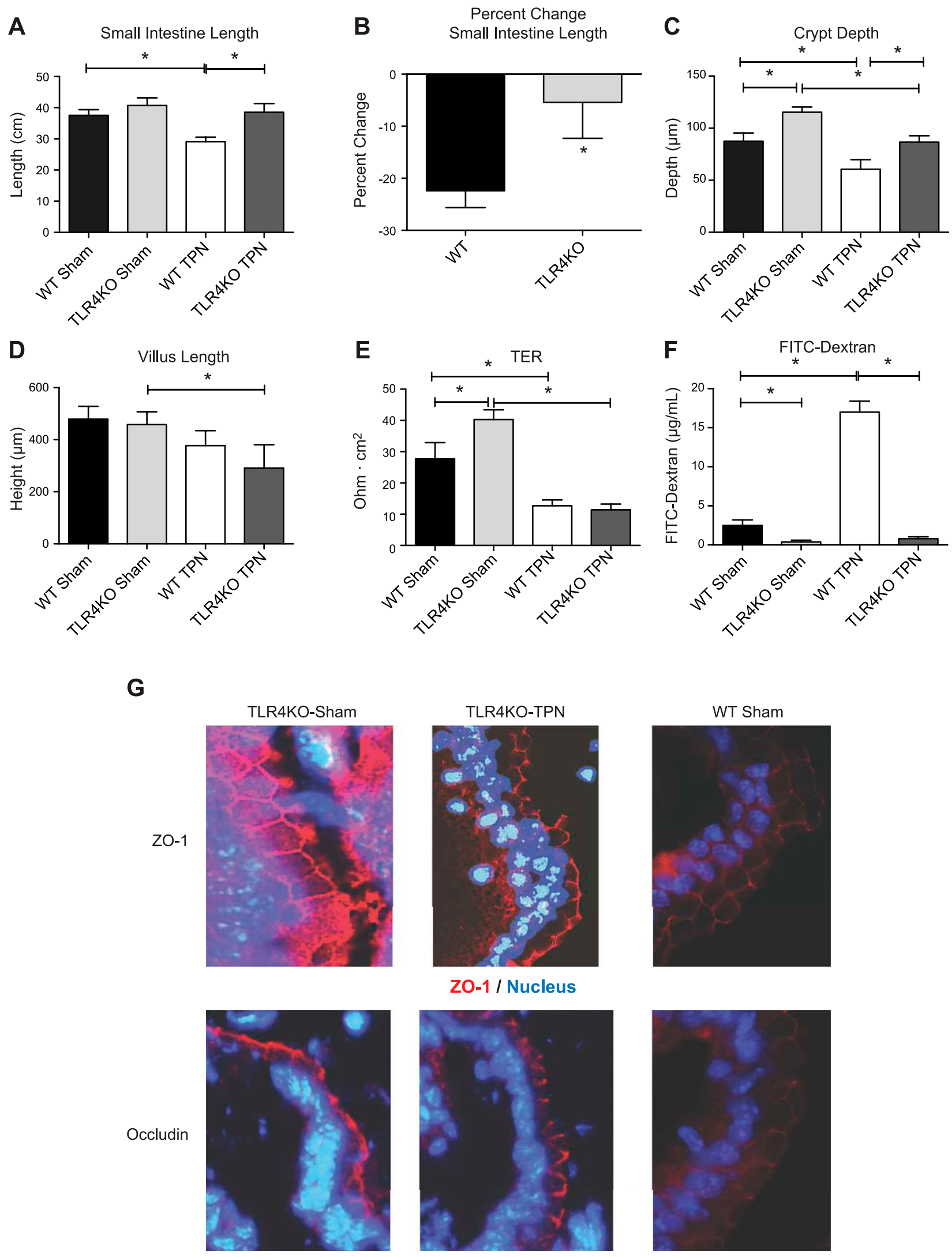

\section{ZO-1 / Nucleus}
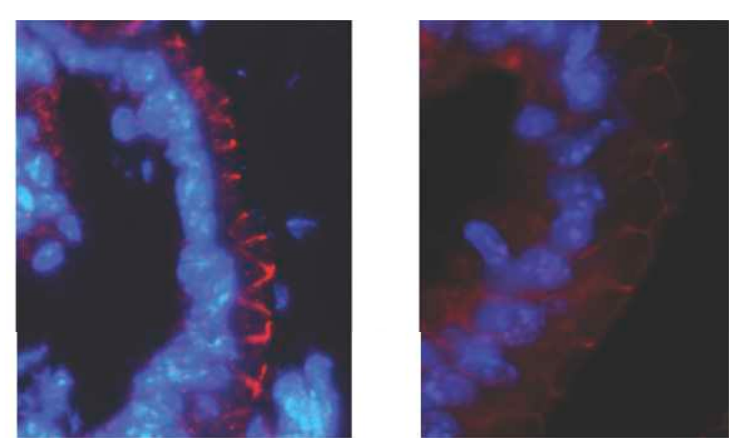

Occludin / Nucleus

Figure 1. Gross and microscopic morphometric measurements of TLR4KO and WT mice. A) Mean small intestine lengths. $B$ ) Percentage change in small intestine length with TPN administration in WT and TLR4KO groups. $C$ ) Differences in crypt depth. $D$ ) Differences in villus length. Results represent means $\pm \mathrm{SD}, n=6$ /group. ${ }^{*} P<0.05$. E) Differences in transepithelial resistance for each study group. $F)$ Permeability of FITC-dextran for each study group. $G$ ) Representative images of ZO-1 and occludin staining for each group. 
compared to WT-TPN mice $(-5.41 \pm 6.9 \%$ vs. $-22.40 \pm 3.3 \%$, respectively; $P<0.0001$; Fig. $1 B$ ).

Although grossly TLR4KO mice did not appear different, when compared microscopically, TLR4KO mice had significantly deeper crypts than WT mice in both the shamtreated (1.3-fold deeper; $P=0.002$; Fig. $1 C$ ) and TPN groups (1.4-fold deeper; $P=0.0004$; Fig. $1 C$ ). Additionally, while TLR4KO mice undergo loss of crypt depth with TPN administration, crypts remain deeper than their WT-TPN counterparts. With respect to villus length (Fig. 1D), TLR4KO-sham mice were similar to WT-sham mice. With TPN administration, TLR4KO mice showed loss of villus length; however, the resultant decline in length was not significantly different from WT-TPN mice. Overall, there was a protection of both intestinal length and crypt depth in a proinflammatory model with TLR4KO.

\section{Enterally fed TLR4KO mice have stronger EBF}

Another consequence of TPN-associated mucosal atrophy is a loss of EBF (24). To address the effect of removing TLR4 signaling in the TPN model, we examined 2 measures of barrier function. For enterally fed groups, it was interesting to note that TLR4KO-sham mice had higher transepithelial resistance compared to WT-sham mice (40.3 \pm 3.1 vs. $27.7 \pm 5.2 \Omega \cdot \mathrm{cm}^{2} ; P=0.003$; Fig. $\left.1 E\right)$. However, this increase in EBF was lost with TPN administration. With TPN, both WT and TLR4KO groups had a similar decline in transepithelial resistance $(11.38 \pm 1.8$ vs. $12.7 \pm 1.8 \Omega \cdot \mathrm{cm}^{2} ; P=0.32$; Fig. $\left.1 E\right)$.

EBF was also measured by permeation of a tracer molecule (FITC-dextran). Permeation was decreased in enterally fed TLR4KO mice, suggesting improved EBF at baseline. Interestingly, the typical 6-fold increase in FITCdextran permeation observed in WT-TPN mice was not observed in the TLR4KO-TPN group (Fig. $1 F$ ). In fact, there was minimal change in permeation between TLR4KO-sham and TLR4KO-TPN groups.

To further examine the structural changes in tight junction integrity, we analyzed IF staining of several junctional proteins, including ZO-1, occludin, and claudin 2. Our laboratory has previously shown a significant decline in both ZO-1 and occludin in WT mice with TPN administration (24). When looking at the TLR4KO mice, there were no significant changes in ZO-1 (Fig. $1 G$ ) and claudin 2 (data not shown) staining between TLR4KO-sham and TLR4KO-TPN groups. Occludin did appear to be slightly decreased in the TLR4KO-TPN group. However, the typical internalization of occludin protein with TPN administration in WT mice was not observed in the TLR4KO group (Fig. 1G).

\section{TLR4KO mice have increased IEC proliferation}

The differences observed in the crypt morphology could represent either an expansion in IEC proliferation or a loss of IEC apoptosis. Therefore, we next examined IEC proliferation rates using BrdU and PCNA staining. WT mice had a significant loss of IEC proliferation with TPN administration as measured by significant declines in both BrdU and PCNA staining $(-18.7 \%$ and $-28.4 \%$, respectively; Fig. 2). Interestingly, this marked decline in IEC proliferation was nearly completely prevented in TLR4KO-TPN mice, and levels of proliferation were not significantly different compared to TLR4KO-sham mice. Thus, maintenance of IEC proliferation in TLR4KO mice was a contributing mechanism preventing TPNassociated atrophy.

\section{TLR4KO have decreased crypt IEC apoptosis rates}

We then examined IEC apoptosis rates using TUNEL staining (Fig. 3A, B). With TPN administration, villi apoptosis rates increased 5-fold in WT mice and almost 10-fold in TLR4KO mice. In WT mice, crypt apoptosis rates increased 1.8-fold with TPN and was similar to levels previously described by our group. However, TLR4KO mice showed very low levels of apoptotic positive crypt cells in both the sham-treated and TPN groups. As a result, apoptotic positive crypt cells were 23-fold higher in WT-TPN than in TLR4KO-TPN mice.

To complement TUNEL staining, caspase 3 staining was also completed. Similar to our TUNEL staining, crypt apoptosis rates were significantly decreased in TLR4KO mice. Interestingly, however, there was a significant increase in villous IEC apoptosis rates with TPN administration for both WT and TLR4KO groups (Fig. 3). This suggested that the regulatory pathways that intersect between TLR4 and EGF signaling may reside within the crypt region of the mucosa.

To account for this significant reduction in crypt cell apoptosis, Bcl-2 (antiapoptotic) and Bax (proapoptotic) expression were then measured in mucosal scrapings at the RNA level (Fig. 4A-C). For sham-treated mice, TLR4KO level of Bcl-2 was 9-fold higher than WT mice $(P=0.003)$; for TPN mice, TLR4KO was 1.6-fold higher than WT mice $(P=0.004$; Fig. 4A). Overall, WT mice showed a 4-fold increase in Bcl-2 with TPN administration, while TLR4KO-TPN mice showed a 1.3-fold decrease. For Bax (Fig. 4B), there was a 10-fold decrease in TLR4KO-TPN compared to the 4-fold increase observed with TPN administration in WT mice.

We next examined the Bax/Bcl-2 ratio (Fig. $4 C$ ), as this ratio may have more physiologic relevance to overall development of apoptosis (25). While sham-treated groups were similar between WT and TLR4KO mice, WT mice showed a 1.7-fold increase in the ratio with TPN administration; however, in TLR4KO-TPN group, we observed a 14-fold decrease in the $\mathrm{Bax} / \mathrm{Bcl}-2$ ratio. As a result, the TLR4KO-TPN ratio was 24-fold lower than the WT-TPN ratio $(P=0.0009)$. Thus, this altered balance of pro-and antiapoptosis factors may contribute to the mechanisms decreasing atrophy of the small intestine with TPN administration.

We have previously described an increase in the extrinsic apoptosis pathway in our TPN model via a 2.5-fold increase in FasL expression (19). In TLR4KO-sham mice, there was a 5.7-fold decrease in FasL compared to WT-sham mice $(0.00044 \pm 0.0003$ vs. $0.0025 \pm 0.0005 ; P=0.015$; Fig. $4 D)$. Although both TLR4KO groups had lower concentrations of Fas than their WT counterparts (Fig. 4E), these differences did not reach significance, and FasL abundances were not significantly different in the TPN groups.

Another downstream pathway that could drive apoptosis is the up-regulation of NF- $\mathrm{B}$ with TPN administration in WT mice (26). Previous research has shown that LPS 

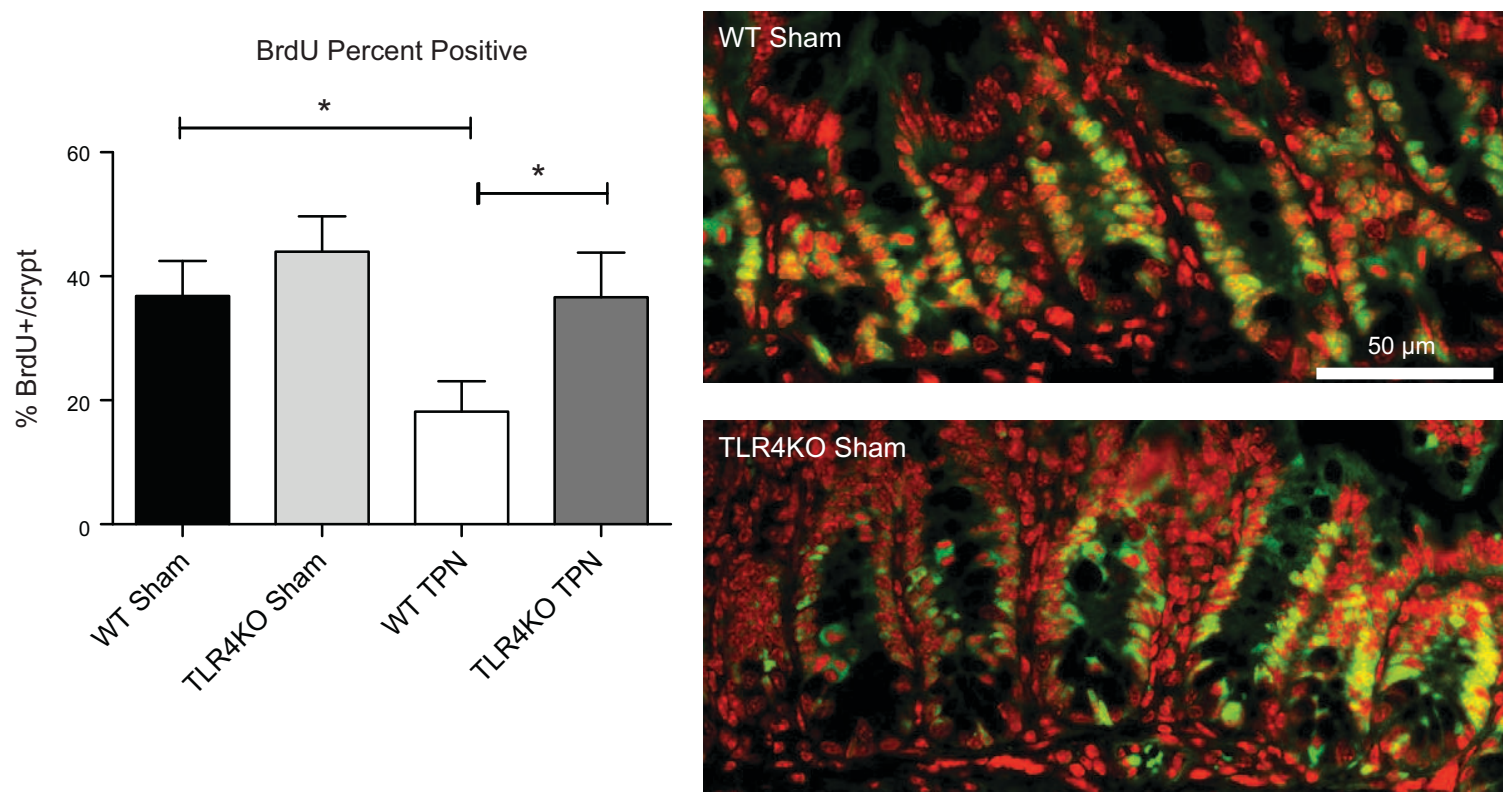

B
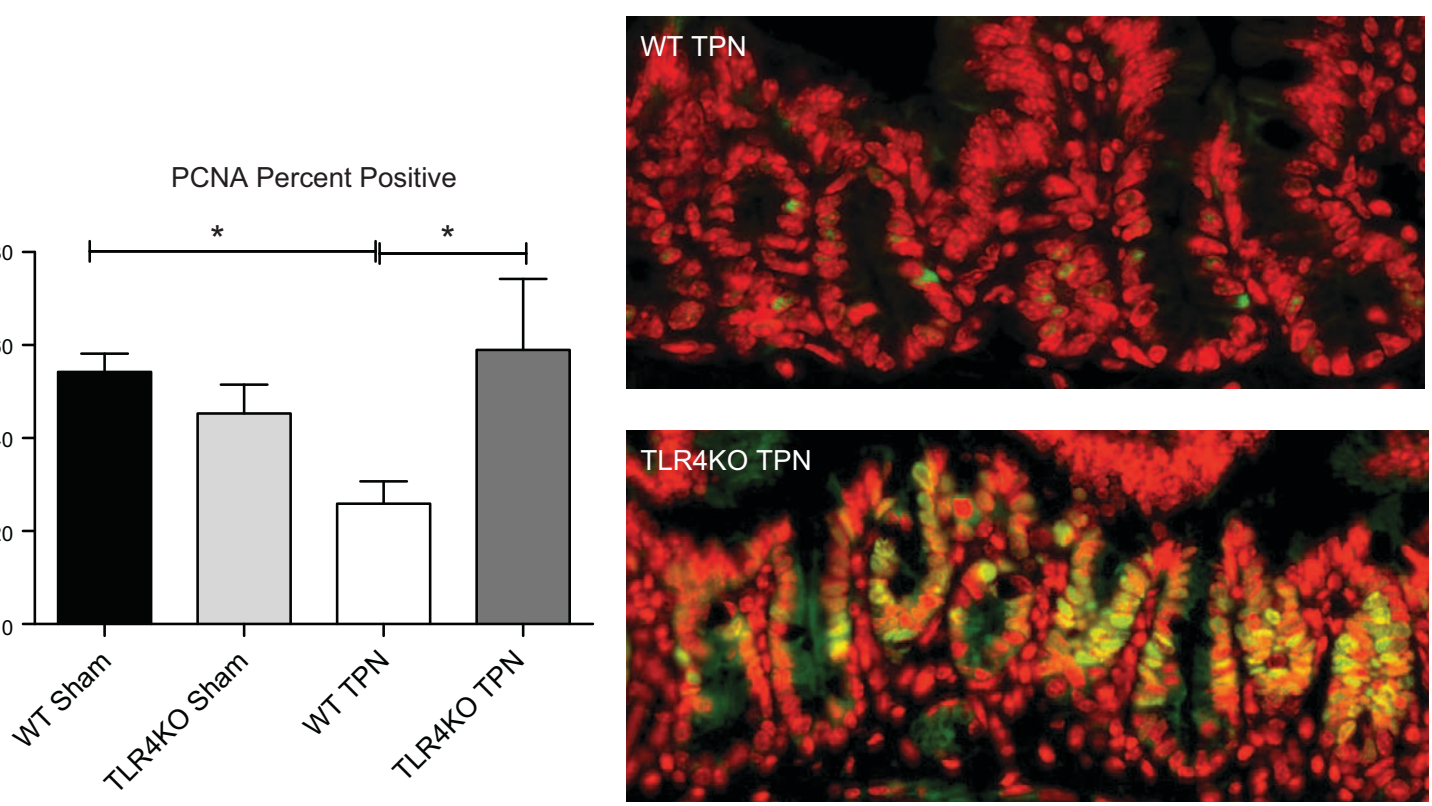

Figure 2. Epithelial cell proliferation is measured between the groups using percentage positive BrdU and PCNA staining. A) Percentage positive BrdU IEC per crypt (mice were injected with BrdU 24 hours before the mice were killed). B) PCNA percentage positive IEC per crypt. C) Representative images of PCNA staining for each study group. Results of (A) and (B) are expressed as means \pm SD of epithelial cell counts from 10 well-oriented crypt-villus complexes from each mouse, $n=6 /$ group. $* P<0.05$.

activates NF-кB through TLR4-dependent and -independent pathways in the colon (27). Interestingly, when comparing phosphorylated Ік $\beta \alpha$ to nonphosphorylated Ік $\beta \alpha$, there was no change in the ratio with TPN administration in TLR4KO mice (Fig. 4F). However, when comparing TLR4KO-TPN and WT-TPN ratios, there was a 1.75-fold increase in this ratio, suggesting a decline in NF- $\mathrm{BB}$ activation in TLR4KO-TPN mice $(P=0.04)$, which is consistent with previously published literature on TLR4KO mice in a dextran sodium sulfate inflammatory model (28). In summary, an overall decrease in apoptosis also contributes to preservation of intestinal length and crypt depth.

\section{TLR4KO have decreased TNF- $\alpha$ but increased IFN- $\gamma$ abundance}

Our laboratory has previously reported a significant increase in the expression of proinflammatory cytokines with the administration of TPN; as we noted above, this is driven by an up-regulation of NF- $\mathrm{BB}$ activation $(6,29)$. Therefore, to look at other possible contributing factors that led to the preservation of proliferation and prevention in apoptosis, we next analyzed changes in mucosal expression of several cytokines. TNF- $\alpha$ increased with TPN administration in WT mice (2-fold over sham-treated group; $P=0.008$; 
A

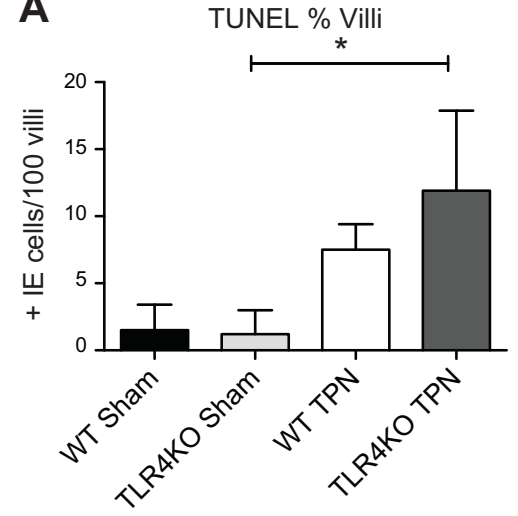

B

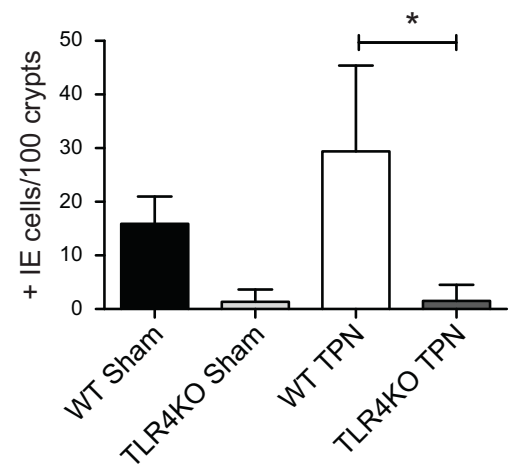

G

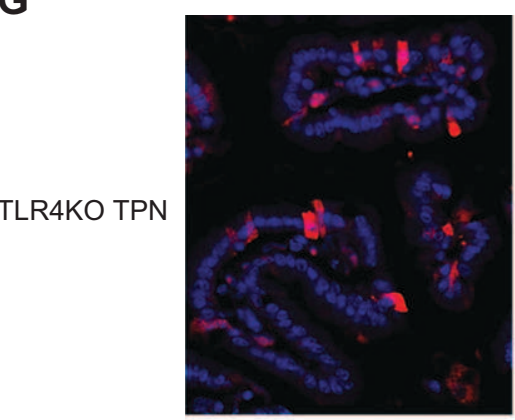

D WT TPN

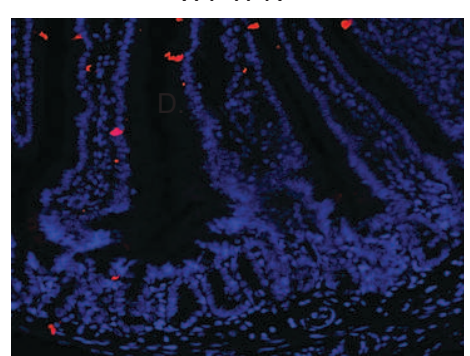

$\mathbf{E}$
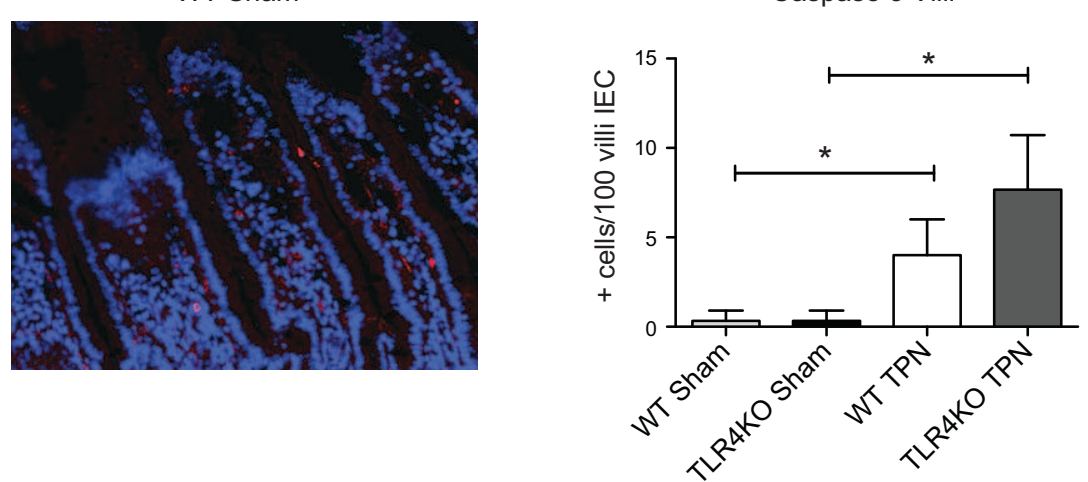

$\mathbf{F}$

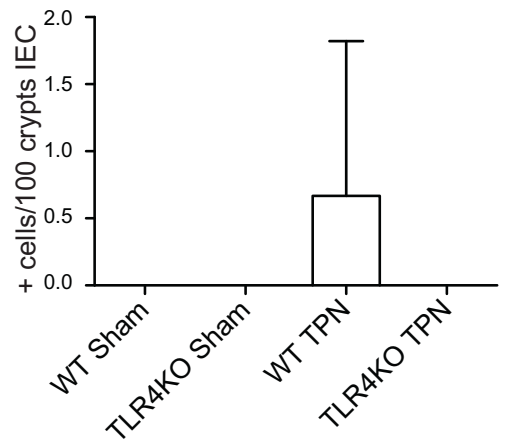

Crypts
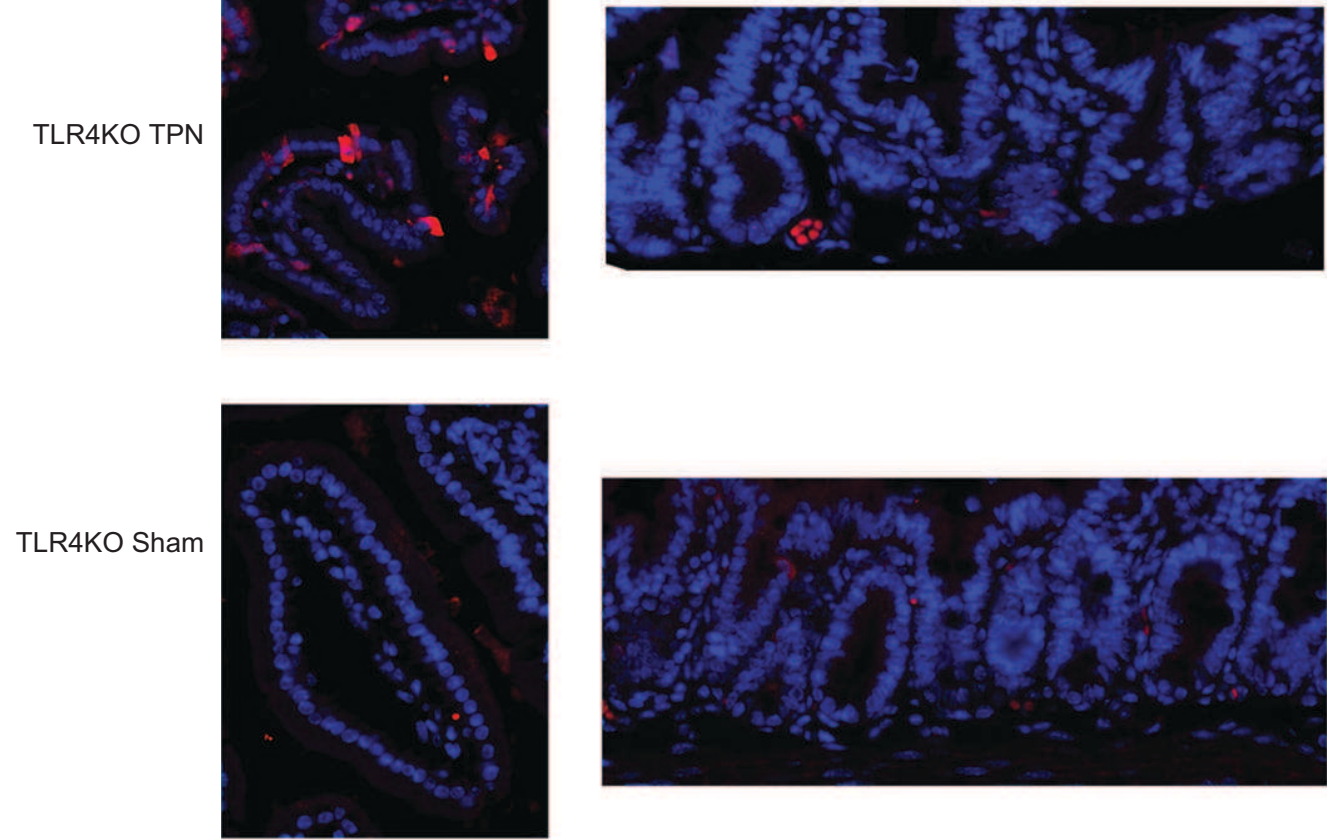

Figure 3. Epithelial cell apoptosis results using TUNEL and caspase 3 staining. A) Mean TUNEL-positive IEC per 100 villi are shown for each study group. $B$ ) Mean TUNEL-positive IEC per 100 crypts are shown for each study group. $C, D$ ) Representative WT images. E) Mean caspase 3 positive per 100 villi IEC are shown for each study group. $F$ ) Mean caspase 3 positive per 100 crypt IEC are shown for each study group. $G$ ) Representative images of caspase 3 staining for each study group. 

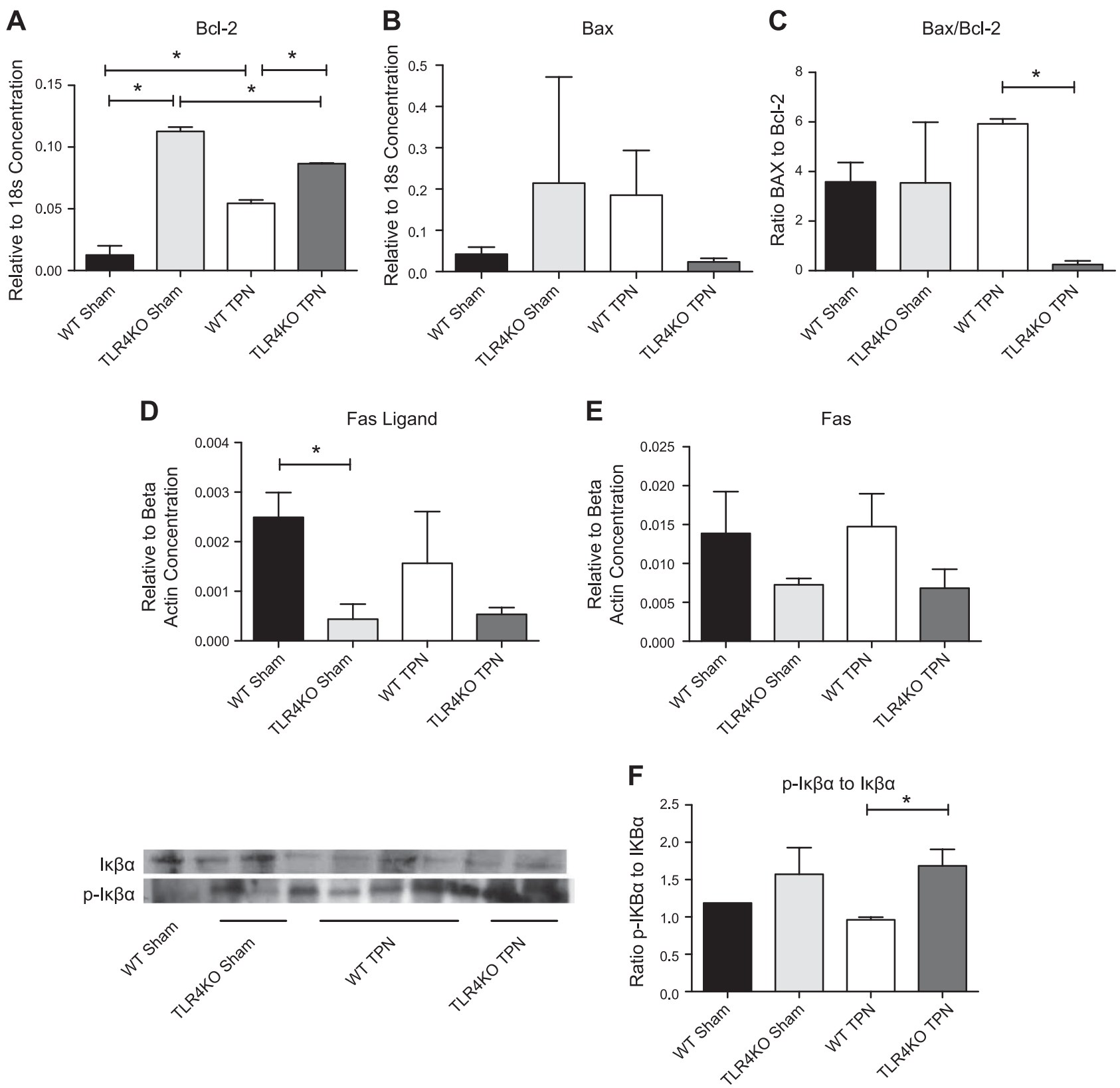

Figure 4. Epithelial cell apoptosis results and contributing apoptotic pathways. $A-C$ ) Factors are measured by RT-PCR of jejunal mucosal scrapings, corrected to expression of $18 \mathrm{~S}$. They represent Bcl-2, Bax, and the Bax/Bcl-2 ratio, respectively. D, E) Fas ligand and Fas mRNA expression adjusted as a ratio to $\beta$-actin expression. $F$ ) Representative Western blot of phosphorylated Iк $\beta \alpha$ to $\mathrm{I \kappa} \beta \alpha$ and ratio of these factors. Results represent means $\pm \mathrm{SD}, n=6$ /group. $* P<0.05$.

Fig. 5A), similar to what we previously reported (6). Despite the decline in NF- $\mathrm{B}$ activation in TLR4KO mice, they showed very little change in TNF- $\alpha$ concentration with TPN administration, and TNF- $\alpha$ levels were similar between TLR4KO-TPN and WT-sham mice.

WT mice showed an increase in both TNFR1 and TNFR2 with TPN administration (Fig. $5 B, C$ ), with only the increase in TNFR2 being significant $(P=0.008$; Fig. $5 C)$. In both TLR4KO groups, we found that abundances of TNFR1 and TNFR2 were both up-regulated to levels similar to those in WT-TPN mice (Fig. 5B, $C$ ).

Interestingly, a marked increase in IFN- $\gamma$ was noted in TLR4KO mice with TPN administration that was not observed in the WT-TPN mice (Fig. $5 D$ ). There were no changes in IL-6 and IL-10 abundances (Fig. $5 E, F$ ).

This suggested that the loss of TLR4 signaling may lead to an up-regulation in other proinflammatory cytokines, potentially via signaling through alternative TLRs. To address this, we then examined expression of other TLRs by RTPCR. This showed a 10-fold increase in TLR2 $(P=0.05$; Fig. 5 Gi) and a 17 -fold increase in TLR5 $(P=0.03$; Fig. 5 Gii $)$ between enterally fed TLR4KO versus WT mice. The increase in TLR2 decreased 3-fold with TPN administration $(P=0.0003$; Fig. 5Gi); however, the TLR5 abundance in TLR4KO-TPN mice decreased to levels similar to WT-TPN mice. No differences in TLR7 abundances were detected 
A

TNF- $\alpha$
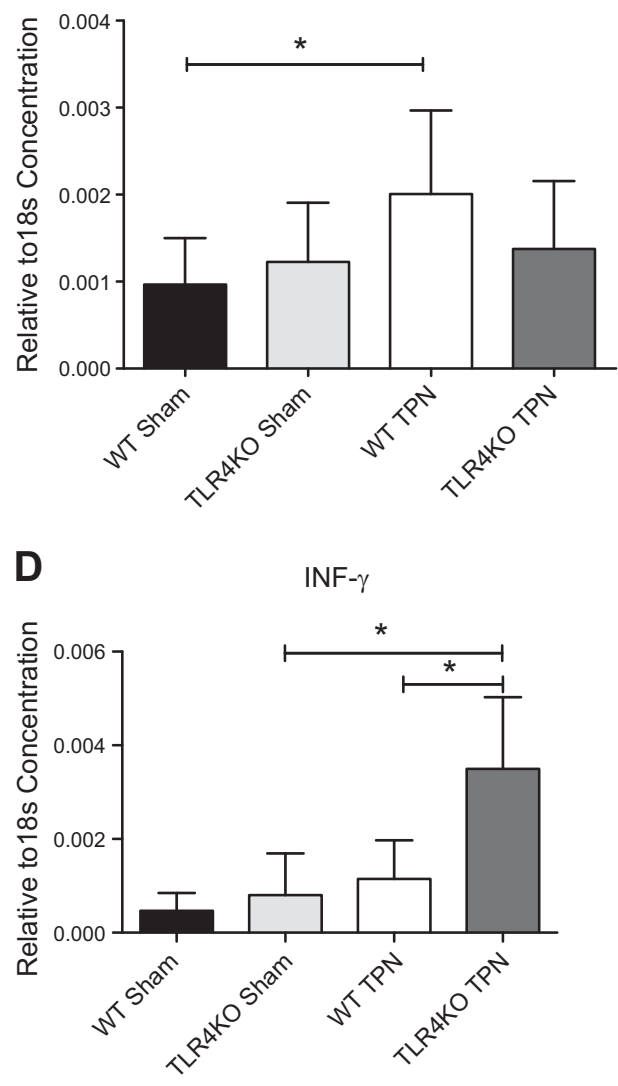

Gi

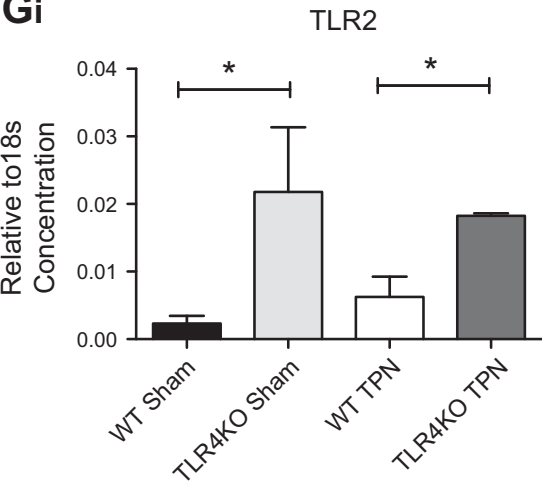

Giii

TLR7

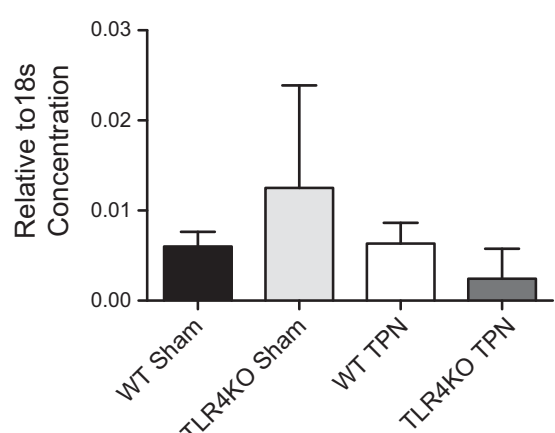

TNFR1

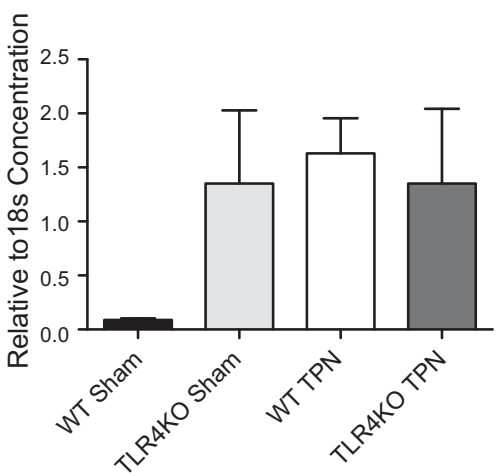

E

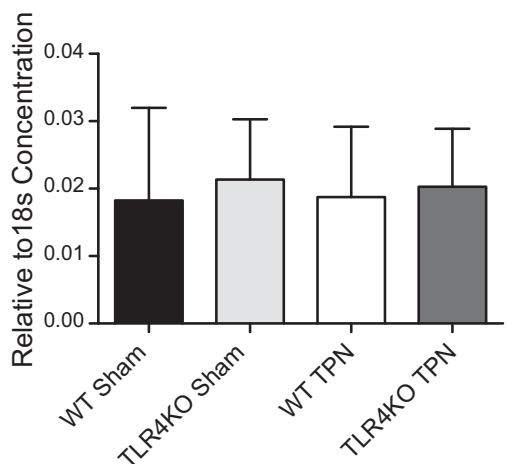

C

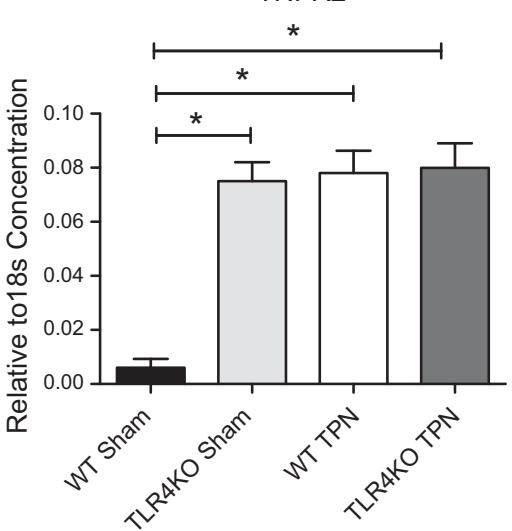

F

$\mathrm{IL}-10$

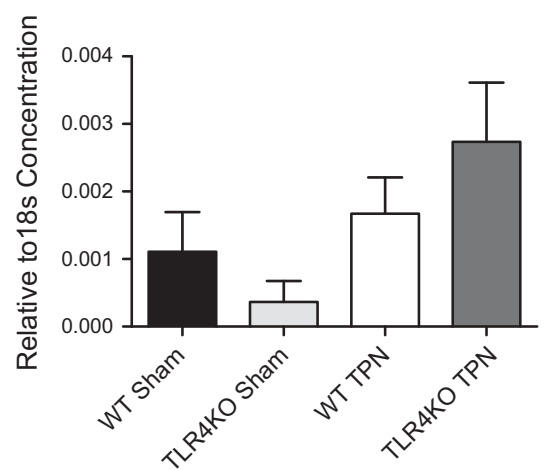

Gii

TLR5

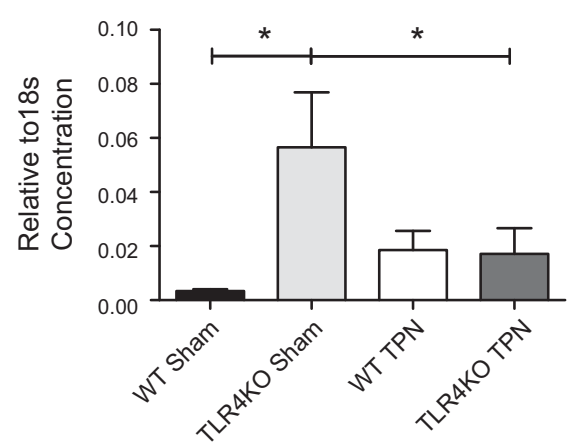

Giv

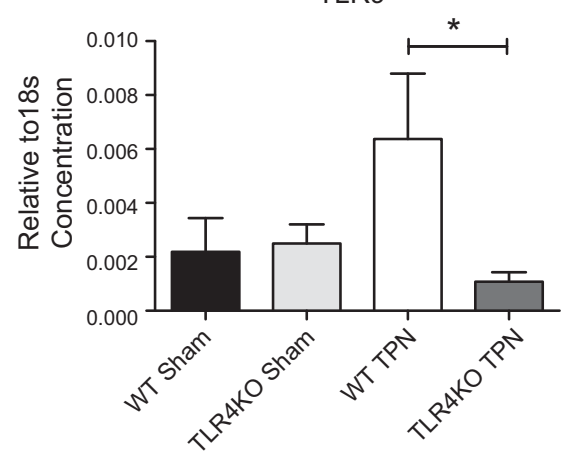

Figure 5. mRNA cytokine expression using RT-PCR of selected cytokines and receptors and TLRs. $A$ ) Changes in TNF- $\alpha$. $B$, $C$ ) Changes in TNFR1 and TNFR2, respectively. $D$ ) changes in IFN- $\gamma . E, F$ ) Changes in IL-6 and IL-10, respectively. Gi-iv) show changes in selected TLRs as a result of the knockout of TLR4 by RT-PCR. mRNA expression is corrected to the expression of $18 \mathrm{~S}$. Results represent means $\pm \mathrm{SD}, n=6$ /group. $* P<0.05$. 
between TLR4KO and WT mice (Fig. 5 Giii). With respect to TLR9, WT mice had an increase in abundance with TPN administration. For the TLR4KO mice, TPN resulted in no significant change in TLR9 abundance. As a result, there was a significant 6 -fold decrease between WT-TPN and TLR4KO-TPN ( $P=0.02$; Fig. 5Giv) .

\section{TLR4KO mice show increased mucosal expression of EGF}

Our laboratory has previously shown a tight interrelation between increases in TNF- $\alpha$ signaling with TPN and a decline in the mucosal expression of EGF (6). We next examined mechanisms that contributed to preserved IEC proliferation in our TLR4KO-TPN mice, as well as whether this preservation of IEC proliferation was due to an interdependency of TLR4 and EGF. Interestingly, mucosal EGF mRNA was significantly increased (26-fold) in TLR4KO-sham versus WTsham mice $(0.032 \pm 0.003$ vs. $0.0012 \pm 0.0003 ; P=0.0006$; Fig. 6A). Although levels declined with TPN in TLR4KO-TPN mice, EGF remained 6 -fold higher in TLR4KO-TPN $v s$. WTTPN mice $(0.020 \pm 0.005$ vs. $0.0033 \pm 0.002 ; P=0.0001$; Fig. $6 A)$. Our laboratory has shown that loss of ErbB1 is a major signaling mechanism that leads to mucosal atrophy in our
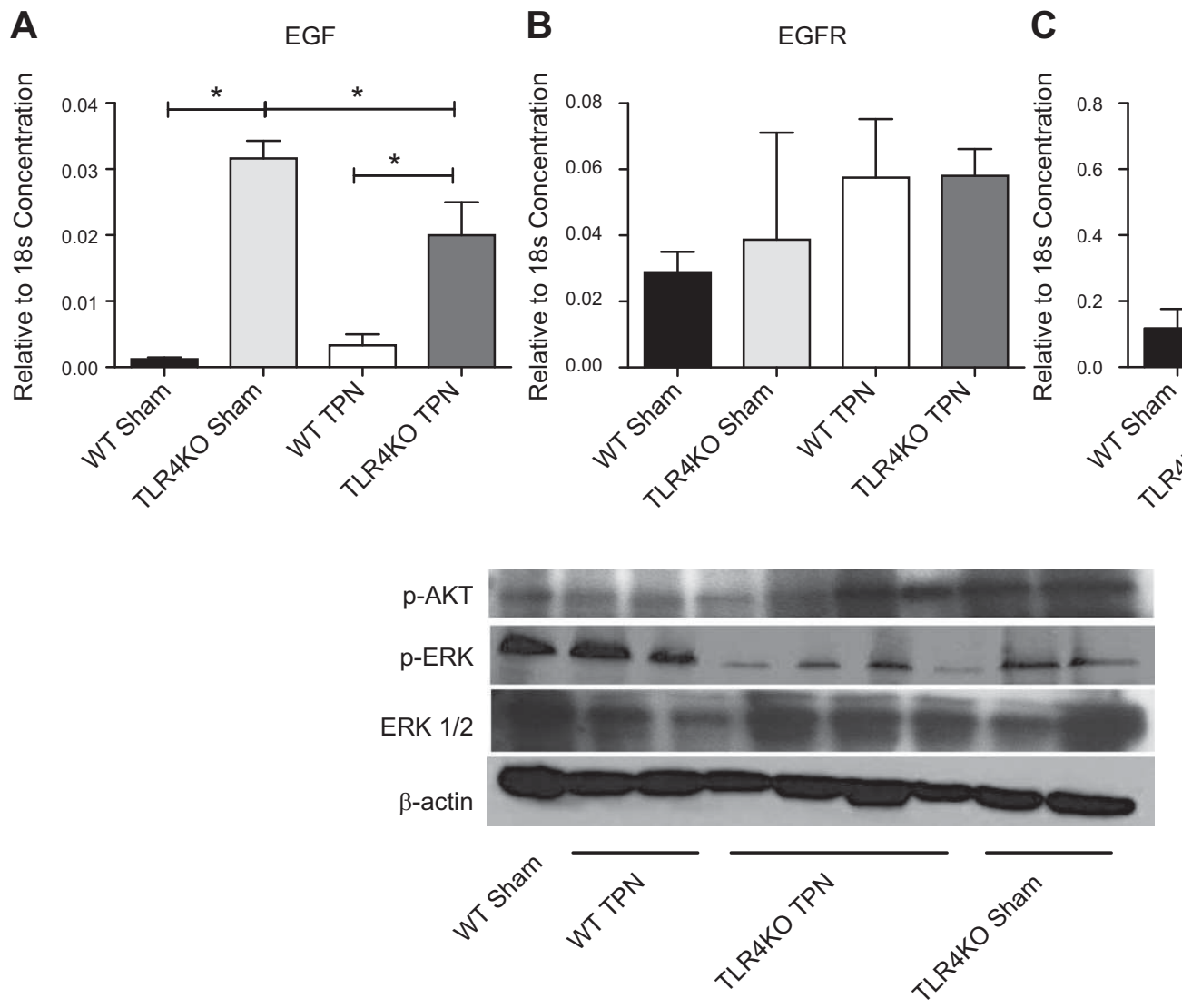

D

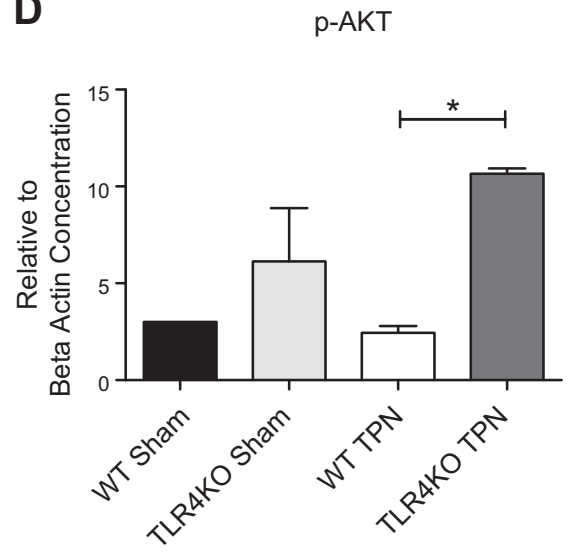

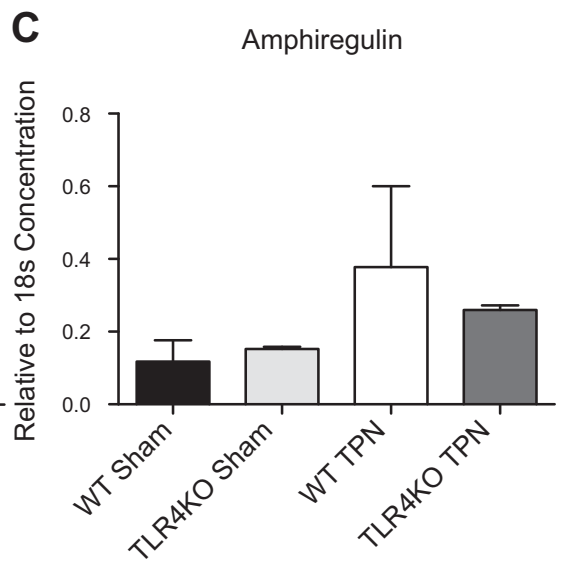

$\mathbf{E}$
p-ERK to ERK ratio

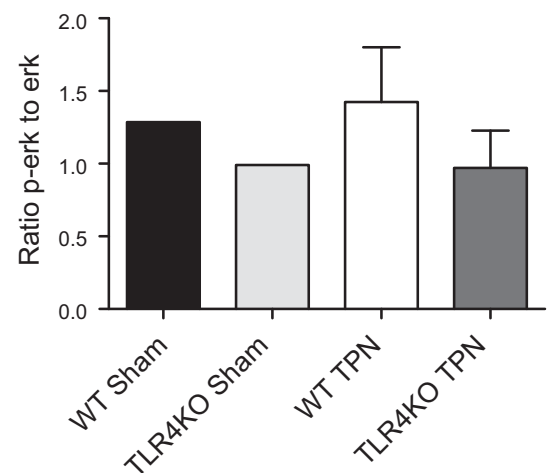

Figure 6. EGF/EGFR and downstream signaling factors. A, B) Changes in EGF and EGFR, respectively, by RT-PCR of jejunal mucosal scrapings. C) Changes in amphiregulin by RT-PCR. $D, E$ ) Representative blots from analysis of Western immunoblots of epithelial cell lysate. D) Ratio of p-AKT to $\beta$-actin. E) Ratio of phosphorylated Erk to Erk. Results represent means \pm sD, $n=$ minimum of 5 /group. $* P<0.05$. 
TPN model (6). The expression of the EGF receptor, ErbB1, however, was not significantly changed between TLR4KO and WT mice (Fig. 6B).

In addition, previous studies have suggested that the ErbB1 ligand amphiregulin is critical for mediating proliferation in a TLR4-dependent pathway (13). Therefore, we examined amphiregulin abundance, but we did not detect any significant changes between groups (Fig. 6C). Other ErbB ligands analyzed (heparin-binding epidermal growth factor [HB-EGF], epiregulin, and neuroregulin [NRG] 1, NRG3, and NRG4) also failed to show any significant changes between study groups.

We next examined several dominant downstream signaling pathways for EGF using immunoblotting. We have previously identified a loss of p-AKT expression with TPN administration $(6,21)$. In addition, previous work had shown that exogenous EGF partially prevented the loss of p-AKT signaling in TPN mice (6). For TLR4KO-TPN mice, there was a 4.4-fold increase in p-AKT compared to WTTPN mice (Fig. 6D). A downstream pathway that is upregulated with TPN administration and may contribute to loss of IEC function is the phosphorylation of ERK (6). In TLR4KO-TPN mice, there was a relative decrease in the phosphorylated Erk/ERK ratio compared to WT-TPN mice (Fig. 6E). Thus, it appears that TLR4KO mice have an up-regulation of EGF abundance and EGF downstream signaling pathways.

\section{EGF blockade prevents rescue from TPN-associated atrophy in TLR4KO mice}

To verify our hypothesis that EGF signaling is augmented in the absence of an intact TLR4 receptor, we blocked the ErbB1 receptor using gefitinib in TLR4KO-sham and TLR4KO-TPN mice. Our laboratory has previously shown that ErbB1 blockade in WT-TPN mice results in a significant decline in mucosal-derived EGF and ErbB1. We also showed an increased mortality from 0 to $30 \%$ with EGF blockade (6). In the present study, gefitinib treatment of TLR4KO-TPN mice resulted in increased mortality (from 0 to 25\%). Gefinitib treated animals appeared hunched and scruffy at time of killing, suggesting a much poorer clinical condition. All TPN groups lost weight with TPN; however, TLR4KO-sham with gefitinib was the only enterally fed group to lose weight. (Fig. 7A).

ErbB1 blockade resulted in a loss of intestinal length in most groups (Fig. 7B). The reduction in intestinal length in WT-TPN mice was not significantly different from that of TLR4KO-TPN mice. When we calculated the percentage loss of intestinal length, it was similar between WT mice provided TPN and both TLR4KO groups provided EGF blockade (Fig. 7C). This suggests that enhanced EGF signaling was responsible for the preservation of intestinal length in TLR4KO-TPN mice.

The increase in crypt depth observed in TLR4KO-sham group was lost with EGF blockade (115.3 \pm 4.95 vs. $70.73 \pm 4.49$; $P<0.0001$; Fig. 7D). In fact, TLR4KO-sham mice treated with EGF blockade had crypts of similar depth to WT-TPN mice. Additionally, TLR4KO-TPN mice treated with gefitinib had the shallowest crypts of all groups. There was a 2.3-fold decrease in crypt depth compared to TLR4KOTPN and a 1.6-fold decrease compared to WT-TPN mice.
EGF blockade resulted in significant decrease in villus height in both TLR4KO-sham (458.1 \pm 49.1 vs. $164.9 \pm 8.8$; nontreated vs. treated; $P<0.0001$; Fig. 7E) and TLR4KO-TPN groups (291.1 \pm 89.8 vs. $97.6 \pm 11.7$; $P<0.0001$; Fig. 7E). Interestingly, villus height in TLR4KO-sham mice provided EGF blockade group (2.3-fold) and TLR4KO-TPN with EGF blockade group (3.9-fold) were both significantly shorter than WT-TPN mice $(P<0.0001$ for both; Fig. $7 E)$.

When staining for junctional proteins, ZO-1 appeared to be minimally affected by the ErbB1 blockade; however, occludin was severely affected, with a major decrease in expression. The ErbB1 blockade TLR4KO-TPN group had disorganized, intracellular staining for occludin (Fig. $7 F$ ).

Proliferation as measured by PCNA staining was significantly decreased in both the TLR4KO-sham $(P=0.007)$ and TLR4KO-TPN $(P<0.0001)$ groups that received ErbB1 blockade compared to their TLR4KO counterparts that did not receive the blockade (Fig. $7 G$ ). In fact, the proliferation rates in both groups were similar to rates observed in WT-TPN mice.

TLR4KO-TPN mice treated with ErbB1 blockade showed a 15-fold increase in apoptotic positive crypt cells compared to TLR4KO-TPN mice (Fig. $7 H$ ). This resulted in an average apoptosis rate similar to WT-TPN mice $(23.0+11.5$ vs. $29.4+15.9 ; P=0.63)$. When examining causes of this increase in apoptosis, a 43-fold decrease in the expression of Bcl-2 was found in TLR4KO-sham gefitinib mice compared to TLR4KO-sham group $(P<0.0001$; Fig. 8A), which was a similar level as we found in the WT-sham group $(P=0.43)$. The TLR4KO-TPN mice treated with EGF blockade also had a 16.8-fold decrease in Bcl-2 mRNA compared to TLR4KO-TPN mice $(P<0.0001$; Fig. $8 A)$ and a 9.3 -fold decrease compared to the WT-TPN group $(P=0.01$; Fig. $8 A$ ). When we analyzed Bax abundance, there was a significant increase in the TLR4KO-sham with EGF blockade compared to both the WT-sham $(0.25 \pm 0.05$ vs. $0.042 \pm 0.02$; $P=0.01$; Fig. $8 B)$ and TLR4KO-sham $(0.25 \pm 0.05 \mathrm{vs}$. $0.035 \pm 0.008 ; P=0.01$; Fig. $8 B$ ) groups. The TLR4KO-TPN with EGF blockade was similar to the TLR4KO-TPN group and 5.3-fold lower than the WT-TPN group (Fig. 8B).

When we analyzed Fas and FasL with ErbB1 blockade, we found a significant increase in FasL in both the TLR4KOsham $(P=0.02)$ and TLR4KO-TPN $(P=0.03$; Fig. $8 C)$ groups. Interestingly, we saw a 4.6-fold increase in Fas abundance in the TLR4KO-TPN mice that received gefitinib compared to the untreated TLR4KO-TPN mice $(0.031 \pm 0.008$ vs. $0.0068 \pm 0.001 ; P=0.02$; Fig. $8 D)$.

The TLR4KO-sham with EGF blockade group experienced a significant increase in TNF- $\alpha$ compared to both WTsham (5.3-fold; $P<0.0001$ ) and TLR4KO-sham (4.3-fold; $P=0.0006$ ) groups (Fig. 8E). Interestingly, TLR4KO-TPN mice treated with gefitinib had similar TNF- $\alpha$ mucosal levels compared to both WT-TPN and TLR4KO-TPN $(P=0.16)$ groups. Because we previously found an increase in IFN- $\gamma$ in the TLR4KO-TPN group, we wanted to examine the effect of both EGF blockade and TPN on IFN- $\gamma$ concentrations. There was no significant difference between the 3 sham-treated groups. In the TLR4KO-TPN with EGF blockade group, we saw an additional 2.0-fold increase in IFN- $\gamma$ concentration compared to the TLR4KO-TPN group $(0.0071 \pm 0.001 \mathrm{vs.}$ $0.0036 \pm 0.001 ; P=0.0002 ;$ Fig. $8 F)$, for a total of a 4.0 -fold increase over the WT-TPN group $(P<0.0001 ;$ Fig. $8 F)$. 

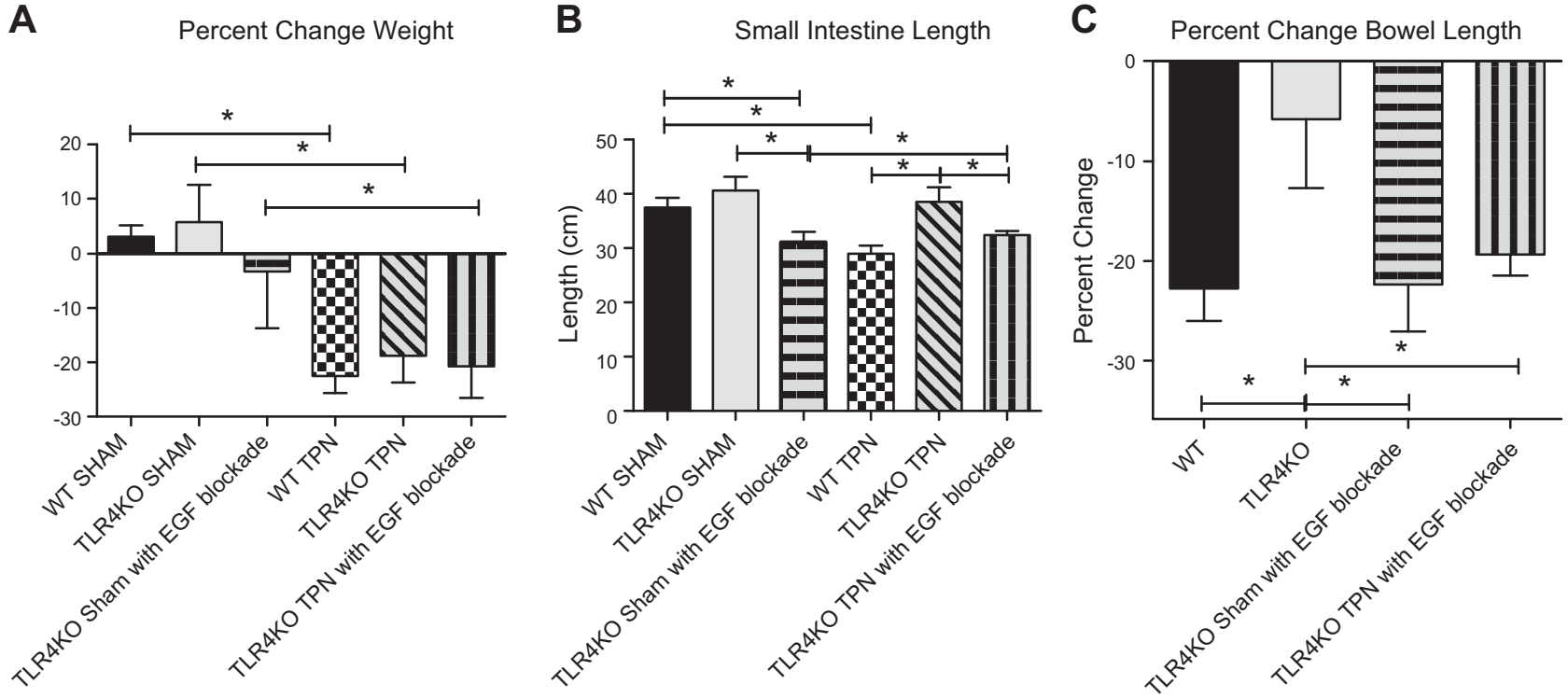

D

$\mathbf{E}$

Villus Length
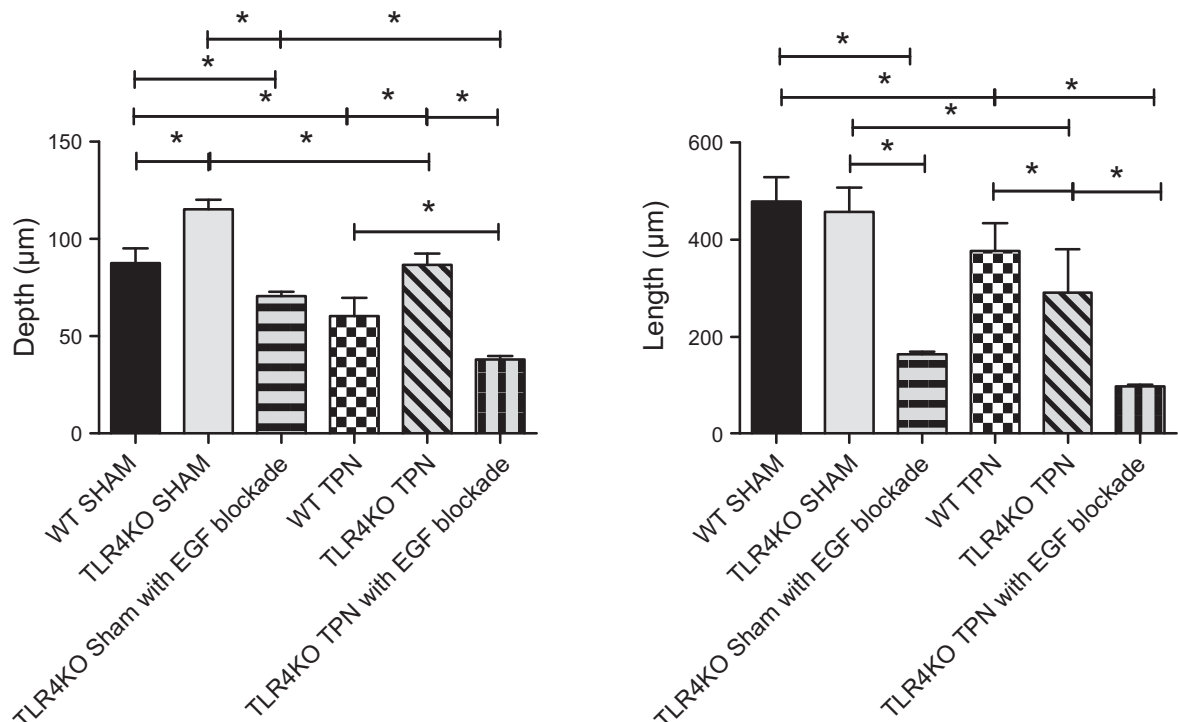

Figure 7. Effect of ErbB1 blockade on the small intestine of TLR4KO mice. A) Percentage change between preoperative and postoperative weights for each group. $B$ ) Changes in small intestine lengths. $C$ ) Percentage change in small intestine length with TPN and ErbB1 blockade. WT is the percentage change in small intestine length between WT-sham and WT-TPN mice. TLR4KO is the percentage change in small intestine length between TLR4KO-sham and TLR4KO-TPN mice. TLR4KO-sham with EGF blockade is the percentage change in small intestine length between TLR4KO-sham and TLR4KO-sham EGF blockade mice. TLR4KO-TPN with EGF blockade is the percentage change in small intestine length between TLR4KO-sham and TLR4KO-TPN EGF blockade mice. $D$ ) Differences in crypt depth for each study group. $E$ ) Differences in villus length for each study group. $F$ ) Representative images of ZO-1 and occludin staining in ErbB1 blockade for TLR4KO sham and TLR4KO TPN groups compared to WT groups. $G$ ) Changes in proliferation by changes in PCNA staining (\% $\mathrm{PCNA}^{+}$crypt cells). H, $I$ ) Changes in apoptosis by TUNEL staining. Results represent means $\pm \mathrm{SD}, n=6$ /group. $* P<0.05$.

Because we identified an increase in abundance of TLR2 with TLR4KO, we also examined the abundance of TLR2 in TLR4KO groups that received ErbB1 blockade. There was no change in TLR2 abundance between TLR4KO-sham and TLR4KO-sham with EGF blockade. However, when comparing TLR4KO-TPN and TLR4KOTPN with EGF blockade, there was a 7.2-fold decrease in TLR2 abundance to an amount similar to WT-TPN mice $(0.024 \pm 0.009$ vs. $0.0034 \pm 0.002 ; P=0.008$; Fig. $8 G)$.
The TLR4KO-sham mice with EGF blockade no longer had an up-regulation of EGF, as seen with the TLR4KOsham counterparts. Their EGF levels were more similar to WT mice (Fig. 8H). TLR4KO-TPN with EGF blockade group had EGF levels similar to both the WT-TPN $(P=0.26)$ and TLR4KO-TPN $(P=0.054)$ groups (Fig. $8 H)$.

In summary, EGF signaling appears to be a dominant mechanism that explains the beneficial effects in IEC physiology in TLR4KO-TPN mice. The data 
$\mathbf{F}$

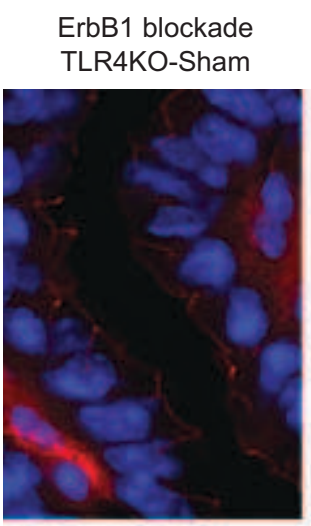

ZO-1

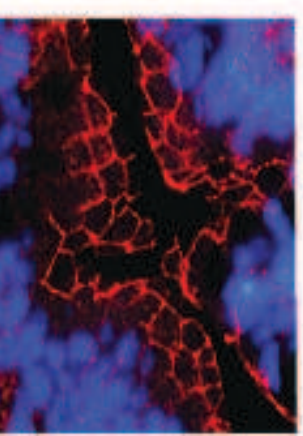

ErbB1 blockade TLR4KO-TPN
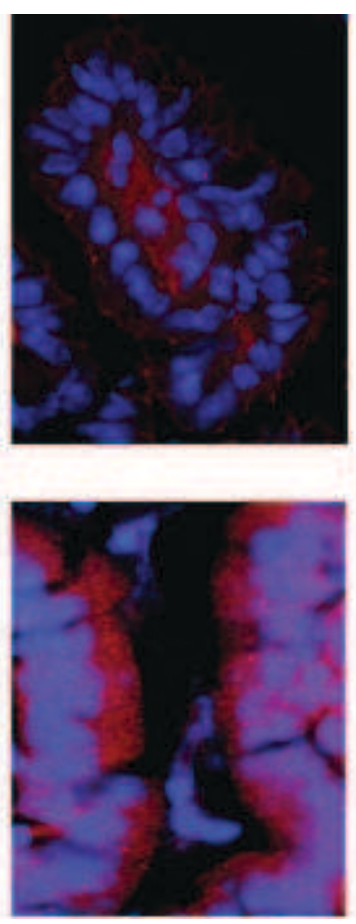

G

PCNA Percent Positive

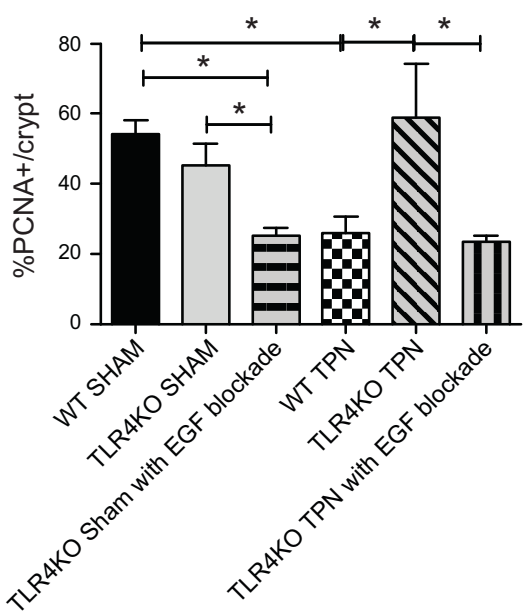

H

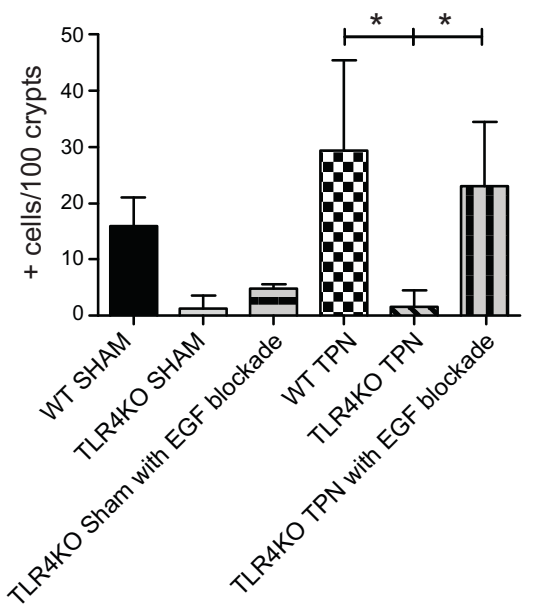

WT

Sham
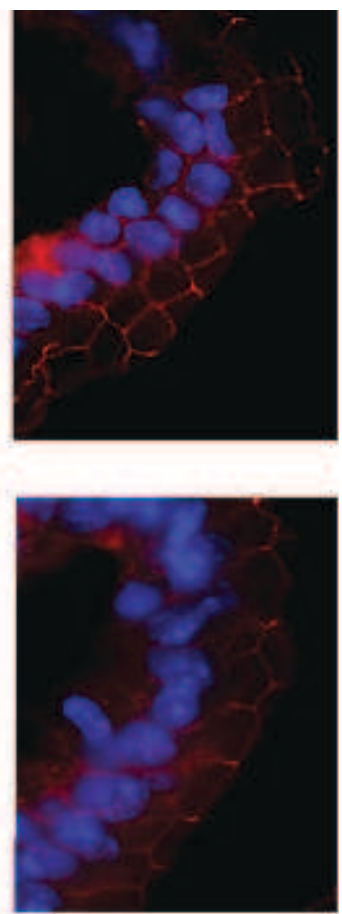

I

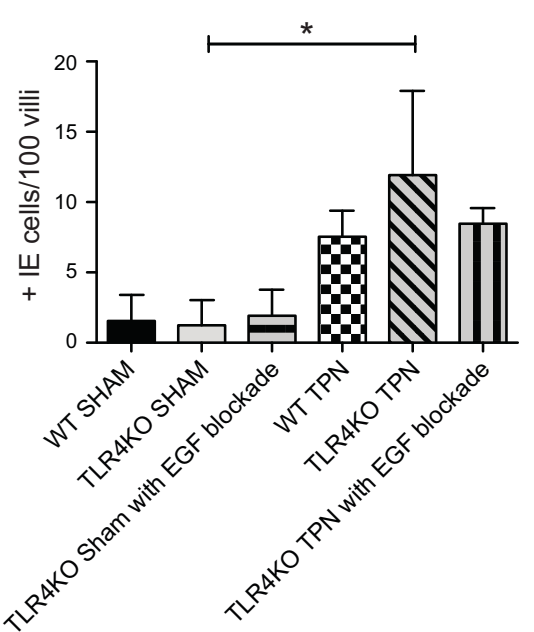

Figure 7. (continued)

also show a strong interdependence between TLR4 signaling and EGF/ErbB1.

\section{DISCUSSION}

This present work demonstrates a strong interdependence of TLR4 and EGF signaling in the remodeling of the crypt/villus architecture during a state of intestinal atrophy driven by enteral nutrient deprivation. Several strikingly novel findings were observed in the present study. Our data show that loss of intact TLR4 signaling within the intestine led to a significant decrease in mucosally derived TNF- $\alpha$ abundance, with a resultant prevention in the loss of EBF and IEC apoptosis. In addition, there was a significant upregulation of EGF expression within the small intestinal mucosa with a resultant increase in IEC proliferation.

Although previous publications have shown decreased inflammation in other proinflammatory models when using the TLR4KO mice (8), TLR4 signaling in the present work led to the novel finding of enhanced IEC growth and prevention of atrophy via enhanced EGF expression. Decreased apoptotic rates in TLR4KO-sham vs. WT-sham were accompanied by unchanged $\mathrm{Bax} / \mathrm{Bcl}-2$ ratio and decreased Fas levels. This is consistent with extrinsic pathway of apoptosis. On the other hand, increased apoptotic rates in WT-TPN versus. WT-sham animals were accompanied by increased Bax/Bcl-2 ratio and unchanged Fas levels. This is 

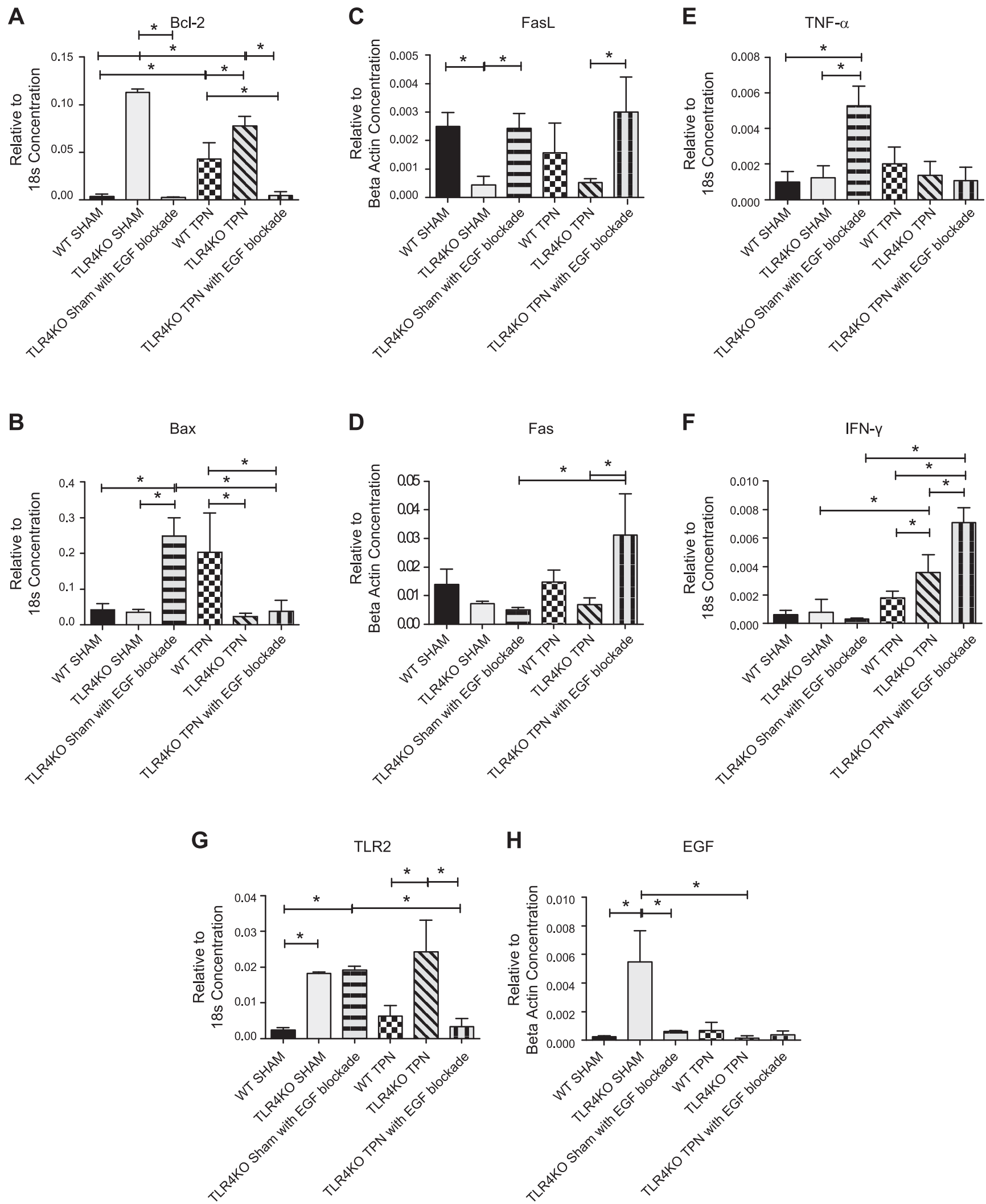

Figure 8. Changes in factors mediating apoptosis and selected cytokines and TLR after ErbB1 blockade in TLR4KO mice; results measured by RT-PCR of jejunal mucosal scrapings. $A, B$ ) Changes in Bcl-2 and Bax, respectively. $C$, $D$ ) Changes in FasL and Fas, respectively, with ErbB1 blockade. $E, F$ ) Changes in TNF- $\alpha$ and IFN- $\gamma$, respectively. $G$ ) Changes in TLR2. H) Changes in EGF. 
consistent with the intrinsic rather than extrinsic pathway being responsible for elevated apoptosis in TPN mice.

The observed decreases in ERK activity also lead to other deductions. TNFR-associated factor 6 (TRAF6) acts as a signal transduction mediator between TLR4 and MAPK pathways (30). TRAF6 can act both dependently and independently of TLR4. Although not specifically measured, TRAF6-dependent and -independent activity would likely have decreased due to the knockout of TLR4, overall observed decline in NF- $\mathrm{KB}$ activation, and observed decrease in ERK phosphorylation. As a result, it is presumed that MAPK activity would also be decreased.

It was interesting that our findings are different from those of Hsu et al., who found a strong dependence on TLR4 signaling and the ErbB1 ligands epiregulin and amphiregulin (13). Using a dextran sodium sulfate colitis model, the expression of both of these ligands were actually decreased in TLR4KO mice, and TLR4 drove amphiregulin and epiregulin expression. In another work from this same laboratory, Sodhi et al. showed that TLR4 signaling promoted IEC proliferation via activation of the same 2 ErbB1 ligands (8). Several differences exist between our current study and the one by Hsu et al. First, we examined small bowel IEC, while their study involved colonic epithelium. Additionally, we noted an increase in mucosal expression of EGF in TLR4KO-TPN mice, which was not reported by Hsu et al. Similar to Hsu et al., we did not see a change in expression of amphiregulin in our TLR4KOTPN mice. Finally, our model of intestinal atrophy was distinctly different than the inflammatory bowel disease model used by this other group of investigators.

We believe that loss of TLR4 signaling in the fed group did not significantly change small bowel characteristics because the majority of the bacterial population is made up of organisms like Bacteroides, which do not signal through TLR4. Fed mice have a small percentage of Gramnegative/LPS-positive bacteria.

Our work also showed that removal of TLR4 partially prevented the loss of EBF in our TPN model. The removal of TLR4 was shown to have a similar protective effect in a neonatal mouse model of necrotizing enterocolitis (31). The mechanisms that drive this protection in this previous study were due to a prevention of enterocyte apoptosis and prevention of mucosal injury. These were similar to the morphologic findings in the current work. In addition, our work suggests that the partial preservation of EBF may well be due to a decline in TNF- $\alpha$, but very possibly also due to enhanced EGF abundance (24). In another study of necrotizing enterocolitis, supplemental growth factors (in this case, heparin-binding EGF) led to a strong preservation of barrier function (32). Such action may be due to the growth factor promoting IEC proliferation and its antiapoptotic actions (9).

It was interesting that TLR4KO mice demonstrated several counterregulatory changes in our TPN model. This included a rise in other TLRs as well as an increase in IFN- $\gamma$. Thus, while blockade of TLR4 had profound protective actions with TPN-associated atrophy, careful examination of the consequences of this blockade is needed. A limitation of the present study was the use of a pan-TLR4KO mouse model. Thus, it is unknown whether blockade of TLR4 on IEC or other intestinal populations (e.g., immunocytes) may be responsible for the findings reported here. While we did not examine the microbiome populations in this current study, we have reported such changes with TPN administration (7). Although subtle changes have been reported with loss of TLR4 signaling, these changes are quite small compared to those seen in our TPN model, and they most likely did not contribute to the altered mucosal growth observed in the current work (33).

Further, the data suggest that an alteration in NF- $\kappa \mathrm{B}$ signaling with TLR4 blockade may play an important role. It was interesting to note that phospho-Ік $\beta \alpha$ was increased in both TLR4KO groups. Recently, a better appreciation that NF- $\mathrm{B}$ may signal via type 1 and 2 pathways (canonical and noncanonical) (34), with a dominant phosphorylation

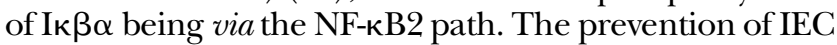
apoptosis in TLR4KO mice suggests a modulation of the NF-кB2 pathway (35); however, this will need to be explored further in future studies.

In conclusion, TLR4 blockade significantly prevented TPN-associated atrophy, potentially via reduction in TNF- $\alpha$ abundance and elevation in EGF levels. Potential clinical applications of such TLR4 blockade may prove potentially useful in patients who are dependent on TPN and who are susceptible to high rates of septicemia resulting from a loss of EBF and who have a decreased ability to tolerate enteral feedings due to intestinal atrophy.

This work was supported by the U.S. National Institutes of Health (NIH) National Institute of Allergy and Infectious Diseases (2R01AI-44076-15), the NIH National Institute of Diabetes and Digestive and Kidney Diseases (5R01DK093697), and the Center for Organogenesis (T32-HD7505). The authors declare no conflicts of interest.

\section{REFERENCES}

1. Braga, M., Ljungqvist, O., Soeters, P., Fearon, K., Weimann, A., and Bozzetti, F.; ESPEN. (2009) ESPEN guidelines on parenteral nutrition: surgery. Clin. Nutr. 28, 378-386

2. Duro, D., Kamin, D., and Duggan, C. (2008) Overview of pediatric short bowel syndrome. J. Pediatr. Gastroenterol. Nutr. 47 (Suppl 1), S33-S36

3. Wildhaber, B. E., Lynn, K. N., Yang, H., and Teitelbaum, D. H. (2002) Total parenteral nutrition-induced apoptosis in mouse intestinal epithelium: regulation by the Bcl-2 protein family. Pediatr. Surg. Int. 18, 570-575

4. Yang, H., Kiristioglu, I., Fan, Y., Forbush, B., Bishop, D. K., Antony, P. A., Zhou, H., and Teitelbaum, D. H. (2002) Interferon-gamma expression by intraepithelial lymphocytes results in a loss of epithelial barrier function in a mouse model of total parenteral nutrition. Ann. Surg. 236, 226-234

5. Kudsk, K. A., Croce, M. A., Fabian, T. C., Minard, G., Tolley, E. A., Poret, H. A., Kuhl, M. R., and Brown, R. O. (1992) Effects of septic morbidity after blunt and penetrating abdominal trauma. Ann. Surg. 215, 503-511

6. Feng, Y., and Teitelbaum, D. H. (2012) Epidermal growth factor/TNF- $\alpha$ transactivation modulates epithelial cell proliferation and apoptosis in a mouse model of parenteral nutrition. Am. J. Physiol. Gastrointest. Liver Physiol. 302, G236-G249

7. Miyasaka, E. A., Feng, Y., Poroyko, V., Falkowski, N. R., Erb-Downward, J., Gillilland III, M. G., Mason, K. L., Huffnagle, G. B., and Teitelbaum, D. H. (2013) Total parenteral nutrition-associated lamina propria inflammation in mice is mediated by a MyD88-dependent mechanism. J. Immunol. 190, 6607-6615

8. Sodhi, C. P., Neal, M. D., Siggers, R., Sho, S., Ma, C., Branca, M. F., Prindle, T., Jr., Russo, A. M., Afrazi, A., Good, M., Brower-Sinning, R., Firek, B., Morowitz, M. J., Ozolek, J. A., Gittes, G. K., Billiar, T. R., and Hackam, D. J. (2012) Intestinal 
epithelial Toll-like receptor 4 regulates goblet cell development and is required for necrotizing enterocolitis in mice. Gastroenterology 143, 708-718, e1-e5

9. Frey, M. R., Edelblum, K. L., Mullane, M. T., Liang, D., and Polk, D. B. (2009) The ErbB4 growth factor receptor is required for colon epithelial cell survival in the presence of TNF. Gastroenterology 136, 217-226

10. Yamaoka, T., Yan, F., Cao, H., Hobbs, S. S., Dise, R. S., Tong, W., and Polk, D. B. (2008) Transactivation of EGF receptor and ErbB2 protects intestinal epithelial cells from TNF-induced apoptosis. Proc. Natl. Acad. Sci. USA 105, 11772-11777

11. Kaiser, G. C., and Polk, D. B. (1997) Tumor necrosis factor alpha regulates proliferation in a mouse intestinal cell line. Gastroenterology 112, 1231-1240

12. McElroy, S. J., Frey, M. R., Yan, F., Edelblum, K. L., Goettel, J. A., John, S., and Polk, D. B. (2008) Tumor necrosis factor inhibits ligand-stimulated EGF receptor activation through a TNF receptor 1-dependent mechanism. Am J Gastrointest Liver Physiol 295, G285-G293

13. Hsu, D., Fukata, M., Hernandez, Y. G., Sotolongo, J. P., Goo, T., Maki, J., Hayes, L. A., Ungaro, R. C., Chen, A., Breglio, K. J., Xu, R., and Abreu, M. T. (2010) Toll-like receptor 4 differentially regulates epidermal growth factor-related growth factors in response to intestinal mucosal injury. Lab. Invest. 90, 1295-1305

14. Fukata, M., and Abreu, M. T. (2007) TLR4 signalling in the intestine in health and disease. Biochem. Soc. Trans. 35, 1473-1478

15. Yang, H., Gumucio, D. L., and Teitelbaum, D. H. (2008) Intestinal specific overexpression of interleukin-7 attenuates the alternation of intestinal intraepithelial lymphocytes after total parenteral nutrition administration. Ann. Surg. 248, 849-856

16. Zhang, C., Feng, Y., Yang, H., Koga, H., and Teitelbaum, D. H. (2009) The bone morphogenetic protein signaling pathway is upregulated in a mouse model of total parenteral nutrition. J. Nutr. 139, 1315-1321

17. Sun, X., Spencer, A. U., Yang, H., Haxhija, E. Q., and Teitelbaum, D. H. (2006) Impact of caloric intake on parenteral nutrition-associated intestinal morphology and mucosal barrier function. JPEN J. Parenter. Enteral Nutr. 30, 474-479

18. Feng, Y., Sun, X., Yang, H., and Teitelbaum, D. H. (2009) Dissociation of E-cadherin and beta-catenin in a mouse model of total parenteral nutrition: a mechanism for the loss of epithelial cell proliferation and villus atrophy. J. Physiol. 587, 641-654

19. Yang, H., Fan, Y., and Teitelbaum, D. H. (2003) Intraepithelial lymphocyte-derived interferon-gamma evokes enterocyte apoptosis with parenteral nutrition in mice. Am. J. Physiol. Gastrointest. Liver Physiol. 284, G629-G637

20. Grossmann, J., Maxson, J. M., Whitacre, C. M., Orosz, D. E., Berger, N. A., Fiocchi, C., and Levine, A. D. (1998) New isolation technique to study apoptosis in human intestinal epithelial cells. Am. J. Pathol. 153, 53-62

21. Feng, Y., McDunn, J. E., and Teitelbaum, D. H. (2010) Decreased phospho-Akt signaling in a mouse model of total parenteral nutrition: a potential mechanism for the development of intestinal mucosal atrophy. Am. J. Physiol. Gastrointest. Liver Physiol. 298, G833-G841

22. Sun, X., Yang, H., Nose, K., Nose, S., Haxhija, E. Q., Koga, H., Feng, Y., and Teitelbaum, D. H. (2008) Decline in intestinal mucosal IL-10 expression and decreased intestinal barrier function in a mouse model of total parenteral nutrition. Am. J. Physiol. Gastrointest. Liver Physiol. 294, G139-G147
23. Krug, S. M., Amasheh, S., Richter, J. F., Milatz, S., Günzel, D., Westphal, J. K., Huber, O., Schulzke, J. D., and Fromm, M. (2009) Tricellulin forms a barrier to macromolecules in tricellular tight junctions without affecting ion permeability. Mol. Biol. Cell 20, 3713-3724

24. Feng, Y., and Teitelbaum, D. H. (2013) Tumour necrosis factor-induced loss of intestinal barrier function requires TNFR1 and TNFR2 signalling in a mouse model of total parenteral nutrition. J. Physiol. 591, 3709-3723

25. Del Poeta, G., Venditti, A., Del Principe, M. I., Maurillo, L., Buccisano, F., Tamburini, A., Cox, M. C., Franchi, A., Bruno, A. Mazzone, C., Panetta, P., Suppo, G., Masi, M., and Amadori, S. (2003) Amount of spontaneous apoptosis detected by Bax/Bcl-2 ratio predicts outcome in acute myeloid leukemia (AML). Blood 101, 2125-2131

26. De Oliveira-Marques, V., Cyrne, L., Marinho, H. S., and Antunes, F. (2007) A quantitative study of NF-kappaB activation by $\mathrm{H}_{2} \mathrm{O}_{2}$ : relevance in inflammation and synergy with TNF-alpha. J. Immunol. 178, 3893-3902

27. Bhattacharyya, S., Dudeja, P. K., and Tobacman, J. K. (2008) Lipopolysaccharide activates NF-kappaB by TLR4-Bcl10dependent and independent pathways in colonic epithelial cells. Am. J. Physiol. Gastrointest. Liver Physiol. 295, G784-G790

28. Fukata, M., Chen, A., Vamadevan, A. S., Cohen, J., Breglio, K., Krishnareddy, S., Hsu, D., Xu, R., Harpaz, N., Dannenberg, A. J., Subbaramaiah, K., Cooper, H. S., Itzkowitz, S. H., and Abreu, M. T. (2007) Toll-like receptor-4 promotes the development of colitisassociated colorectal tumors. Gastroenterology 133, 1869-1881

29. Wildhaber, B. E., Yang, H., and Teitelbaum, D. H. (2003) Total parenteral nutrition-induced apoptosis in mouse intestinal epithelium: modulation by keratinocyte growth factor. J. Surg. Res. 112, 144-151

30. Akira, S., and Takeda, K. (2004) Toll-like receptor signalling. Nat. Rev. Immunol. 4, 499-511

31. Leaphart, C. L., Cavallo, J., Gribar, S. C., Cetin, S., Li, J., Branca, M. F., Dubowski, T. D., Sodhi, C. P., and Hackam, D. J. (2007) A critical role for TLR4 in the pathogenesis of necrotizing enterocolitis by modulating intestinal injury and repair. J. Immunol. 179, 4808-4820

32. Yu, X., Radulescu, A., Zorko, N., and Besner, G. E. (2009) Heparin-binding EGF-like growth factor increases intestinal microvascular blood flow in necrotizing enterocolitis. Gastroenterology 137, 221-230

33. Frantz, A. L., Rogier, E. W., Weber, C. R., Shen, L., Cohen, D. A., Fenton, L. A., Bruno, M. E., and Kaetzel, C. S. (2012) Targeted deletion of MyD88 in intestinal epithelial cells results in compromised antibacterial immunity associated with downregulation of polymeric immunoglobulin receptor, mucin2, and antibacterial peptides. Mucosal Immunol. 5, 501-512

34. Sun, S. C. (2012) The noncanonical NF-кB pathway. Immunol. Rev. 246, 125-140

35. Williams, J. M., Duckworth, C. A., Watson, A. J., Frey, M. R., Miguel, J. C., Burkitt, M. D., Sutton, R., Hughes, K. R., Hall, L. J., Caamaño, J. H., Campbell, B. J., and Pritchard, D. M. (2013) A mouse model of pathological small intestinal epithelial cell apoptosis and shedding induced by systemic administration of lipopolysaccharide. Dis. Model. Mech. 6, 1388-1399

Received for publication December 17, 2014. Accepted for publication March 2, 2015. 